\title{
A DUF-246 family glycosyltransferase-like gene affects male fertility and the biosynthesis of pectic arabinogalactans
}

Solomon Stonebloom,2, Berit Ebert ${ }^{1,2}$, Guangyan Xiong ${ }^{3,4}$, Sivakumar Pattathil ${ }^{5,6}$, Devon Birdseye ${ }^{1}$, Jeemeng Lao ${ }^{1}$, Markus Pauly ${ }^{3,4}$, Michael G. Hahn ${ }^{5,6,7}$, Joshua L. Heazlewood ${ }^{1,8}$ and Henrik Vibe Scheller ${ }^{1,4^{*}}$

\begin{abstract}
Background: Pectins are a group of structurally complex plant cell wall polysaccharides whose biosynthesis and function remain poorly understood. The pectic polysaccharide rhamnogalacturonan-I (RG-I) has two types of arabinogalactan side chains, type-l and type-II arabinogalactans. To date few enzymes involved in the biosynthesis of pectin have been described. Here we report the identification of a highly conserved putative glycosyltransferase encoding gene, Pectic ArabinoGalactan synthesis-Related (PAGR), affecting the biosynthesis of RG-I arabinogalactans and critical for pollen tube growth.

Results: T-DNA insertions in PAGR were identified in Arabidopsis thaliana and were found to segregate at a 1:1 ratio of heterozygotes to wild type. We were unable to isolate homozygous pagr mutants as pagr mutant alleles were not transmitted via pollen. In vitro pollen germination assays revealed reduced rates of pollen tube formation in pollen from pagr heterozygotes. To characterize a loss-of-function phenotype for PAGR, the Nicotiana benthamiana orthologs, NbPAGR-A and B, were transiently silenced using Virus Induced Gene Silencing. NbPAGR-silenced plants exhibited reduced internode and petiole expansion. Cell wall materials from NbPAGR-silenced plants had reduced galactose content compared to the control. Immunological and linkage analyses support that RG-I has reduced type-I arabinogalactan content and reduced branching of the RG-I backbone in NbPAGR-silenced plants. Arabidopsis lines overexpressing PAGR exhibit pleiotropic developmental phenotypes and the loss of apical dominance as well as an increase in RG-I type-II arabinogalactan content.
\end{abstract}

Conclusions: Together, results support a function for PAGR in the biosynthesis of RG-I arabinogalactans and illustrate the essential roles of these polysaccharides in vegetative and reproductive plant growth.

Keywords: Arabidopsis thaliana, Nicotiana benthamiana, Cell wall, Rhamnogalacturonan-l, Pectin, Pollen tube

\section{Background}

Pectins are an important group of structural cell wall polysaccharides in plants and are major constituents of primary cell walls. Pectins are also important components of many foods and food products. Along with the hemicelluloses, pectins form a matrix into which cellulose microfibrils are embedded. Pectins comprise several types of acidic polysaccharide domains: homogalacturonan,

\footnotetext{
* Correspondence: hscheller@lbl.gov

'Joint BioEnergy Institute and Biological Systems and Engineering, Lawrence Berkeley National Laboratory, Berkeley, CA 94720, USA

${ }^{4}$ Department of Plant and Microbial Biology, University of California, Berkeley, CA 94720, USA

Full list of author information is available at the end of the article
}

xylogalacturonan, rhamnogalacturonan-I (RG-I) and rhamnogalacturonan-II (RG-II), that may be interconnected to one another as well as to other polysaccharides and glycoproteins including hemicelluloses and arabinogalactan proteins (AGPs) $[1,2]$. Homogalacturonan consists of linear chains of $\boldsymbol{\alpha}$-1-4-linked D-galacturonic acid residues and can account for $60 \%$ or more of pectin. Galacturonic acid residues are methyl esterified to varying degrees and can be acetylated at $\mathrm{O}-2$ or $\mathrm{O}-3$ in both homogalacturonan and RG-I. In some plants or tissues homogalacturonan is substituted with xylose or apiose making xylogalacturonan and apiogalacturonan, respectively. RG-I (diagrammed in Fig. 1a) is a distinct 


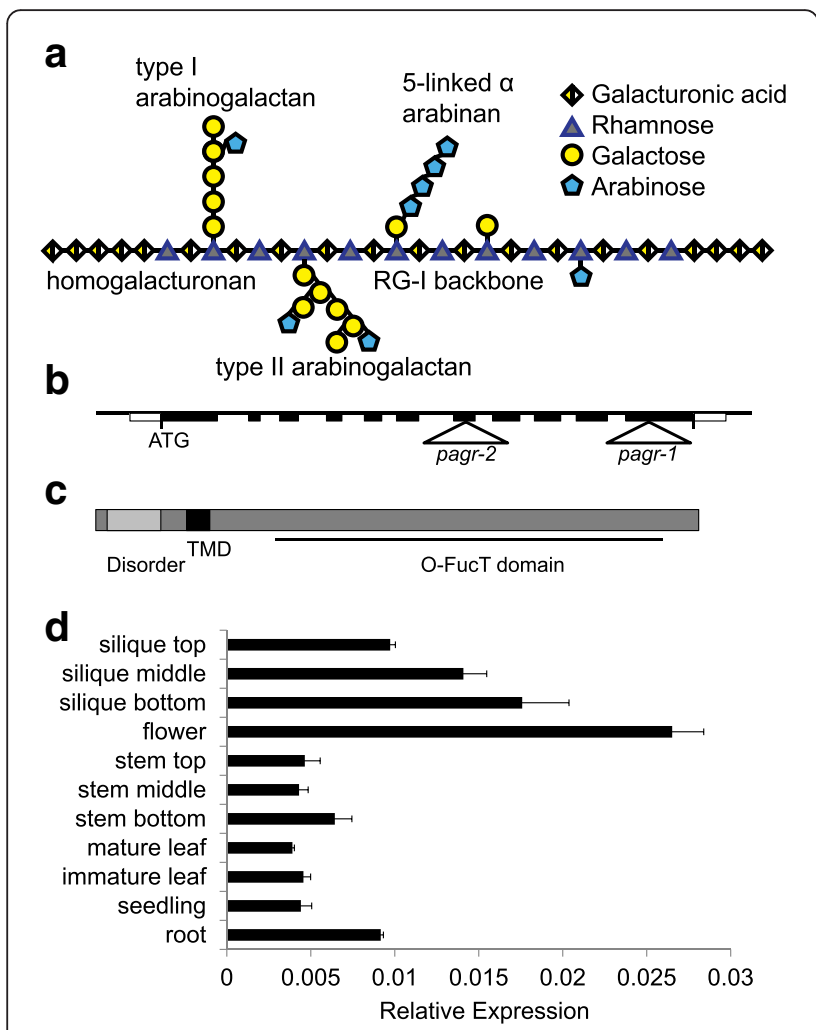

Fig. 1 Schematic representation of the pectic polysaccharide rhamnogalacturonan-I (a) and the structure and expression of PAGR (b-d). a Rhamnogalacturonan-l has a backbone consisting of repeating [-a-D-GalpA-1,2-a-L-Rhap-1,4-] disaccharide units. Approximately half of the rhamnose residues are substituted with sidechains on the 4-position such as type-l and type-II arabinogalactans, $\mathrm{a}-(1,5)$-linked arabinans or single arabinose or galactose residues. b pagr-1 is a T-DNA insertion in the $11^{\text {th }}$ exon of PAGR; pagr-2 is an insertion in the $7^{\text {th }}$ exon. c PAGR encodes a type-II membrane protein with an $\mathrm{N}$-terminal disordered domain and a C-terminal O-fucosyltransferase-like DUF246 domain. d Quantitative RT-PCR analysis of PAGR expression in various Arabidopsis tissues. Results show PAGR expression is highest in reproductive tissues and roots. Error bars indicate standard deviation, $n=3$

class of pectic polysaccharides with a backbone consisting of repeating [- $\alpha-\mathrm{D}-\mathrm{Gal} p \mathrm{~A}-1,2-\alpha-\mathrm{L}-\mathrm{Rha} p-1,4-]$ disaccharide units [3]. Approximately half of the rhamnose residues are elaborated with sidechains on the 4-position, including $\beta$-1,4-linked D-Gal $p$ chains, $\alpha$-(1,5)-linked L-Araf chains as well as type I and IIarabinogalactans. Type-I arabinogalactans consist of $\beta$-1,4-linked D-Gal $p$ backbones with arabinan sidechains, while type-II arabinogalactans possess a backbone of $\beta-1,3-, \beta-1,6$-and $\beta-1,3,6$ - linked D-Gal $p$ similar to the arabinogalactans of AGPs. Rhamnogalacturonan-II is a form of homogalacturonan substituted with four characteristic types of elaborate side chains with distinctive structures made up of 13 different types of monosaccharides and containing the rare sugars L-Aceric acid,
D-Apiose, 2-keto-3-deoxy-D-Lyxo-heptulosaric acid (Dha) and 2-keto-3-deoxy-D-Manno-octulosonic acid (Kdo) $[4,5]$. RG-II forms dimers through boron di-ester bonds and is a ubiquitous component of plant cell walls, highlighting its importance. Given its structural complexity, as many as 67 different transferase activities are thought to be necessary for the biosynthesis of pectin [1].

Plant cell wall polysaccharides are mainly synthesized by glycosyltransferases, enzymes that transfer monosaccharides from an activated donor substrate, usually a nucleotide sugar, onto acceptor molecules, forming glycosidic bonds. Most glycosyltransferases exhibit strong selectivity for donor sugar and acceptor substrates [6]. Glycosyltransferases are classified as "inverting" or "retaining" enzymes depending on whether glycosylation occurs with retention or inversion of stereochemistry at the anomeric carbon atom of the donor substrate. These enzymes have been further classified into families on the basis of amino acid sequence similarities in the Carbohydrate Active enZyme database (CAZy) [7]. The Arabidopsis genome contains 463 genes classified into 41 distinct glycosyltransferase gene families and an additional 100 glycosyltransferase-like genes possessing insufficient similarity to characterized enzymes to be assigned to a CAZy gene family.

To date, only a few enzymes involved in the biosynthesis of pectin have been characterized. These include GAUT1, which is a homogalacturonan galacturonosyltransferase in glycosyltransferase (GT) family 8 [8]. Another GT family 8 member, GATL5, has been shown to be involved in the synthesis of Arabidopsis seed coat mucilage, which is largely composed of RG-I, though its precise role in mucilage biosynthesis has not been established [9]. Xylosyltransferases found in GT family 77, RhamnoGalacturonan-II XylosylTransferase-1 (RGXT1), RGXT2 and RGXT3, have been shown to xylosylate L-fucose in the A-chain of RG-II $[10,11]$. ARAD1 and 2 belong to GT family 47 and are involved in the biosynthesis of arabinans on RG-I; however their catalytic activity has not been demonstrated [12, 13]. XGD1 also belongs to GT family 47 and is a xylosyltransferase that adds xylose to homogalacturonan to form xylogalacturonan [14]. A recently characterized galactan synthase, GALS1, in GT family 92 extends pectic $\beta-1,4$-galactan [15]. GALS1 requires at least a galactotetraose oligosaccharide as a substrate indicating that it extends but does not initiate RG-I galactan biosynthesis. Thus, additional unidentified enzymes are apparently required for the initial branching of RG-I. A recent study suggests that at least some RG-I may be produced as a proteoglycan attached to AGPpolysaccharides [16].

The structure and composition of pectins are altered during growth, development and in response to changing 
conditions. RG-I has specific roles in many plant organs and tissues, and is present in all primary plant cell walls [17]. RG-I is likely present in the cell walls of all vascular plants and has been detected in the walls of basal land plants such as Physcomitrella patens as well as the inner cell wall of the Charophyte alga Penium magaritaceum [18]. Homogalacturonan plays a critical role in the walls of tip growing cells such as root hairs and pollen tubes where de-methyl esterification and cross-linking of homogalacturonan at the edge of the growing tip is thought to solidify the nascent cell wall [19]. RG-I arabinogalactans are also critical components of the pollen tube cell wall. In olive plants pectic galactan forms a ring around the aperture where pollen tubes emerge from the pollen grain [20]. Pectic arabinans likely present as sidechains on RG-I are a critical part of the pollen cell wall [21].

A better understanding of the biosynthesis and processing of pectins is critical to elucidating how this enigmatic class of polymers functions in regulating properties of the plant cell wall. Many uncharacterized GT activities are required for the biosynthesis of pectic polysaccharides. Here we report the identification of a new gene affecting pollen tube growth and present evidence that it is involved in the biosynthesis of pectic arabinogalactans attached to RG-I. We have named the Arabidopsis gene, At3g26370, Pectic ArabinoGalactan synthesis-Related (PAGR).

\section{Results}

PAGR is a highly conserved DUF-246 domain containing protein

Land plants have an expanded group of genes encoding proteins related to GT family 65 , the DUF246 family of GT65-like proteins with 39 members in Arabidopsis [22]. The mammalian GT family 65 protein POFUT1 fucosylates serine/threonine in epidermal growth factor repeats [23]. As the genes that contain a DUF246 domain are an expanded family in plants, some of them are likely to be involved in plant-specific processes [22]. The only previously studied plant DUF246-encoding genes are MSR1 and MSR2, which affect the production and secretion of mannans in Arabidopsis; however their specific role in mannan biosynthesis remains elusive [24]. In examining the predicted amino acid sequences of plant DUF246-containing proteins we identified a gene, At3g26370 (PAGR), encoding a protein that is more highly conserved throughout the land plants than other DUF246 containing proteins. The Selaginella moellendorffii and Physcomitrella patens orthologs of PAGR are $76.0 \%$ and $70.9 \%$ identical to the Arabidopsis protein, respectively (Additional file 1: Figure S1). Other Arabidopsis DUF246 proteins have basal land plant orthologs with pairwise amino acid identities between 45 and $67.7 \%$. The strong conservation of PAGR throughout the land plants can be observed as the short branch length within the PAGR clade in phylogenetic analysis of the DUF246 containing proteins in Arabidopsis, Selaginella and Physcomitrella (Additional file 2: Figure S2). PAGR is predicted to encode a type-II membrane protein with an $\mathrm{N}$-terminal disordered domain, a transmembrane domain and the highly conserved DUF246 domain (Fig. 1c).

\section{PAGR is expressed ubiquitously}

We examined the expression pattern of PAGR in Arabidopsis using quantitative RT-PCR of RNA prepared from various tissues (Fig. 1d). PAGR was expressed in all tissues tested with higher levels of transcript detected in reproductive tissues and roots. Transcript levels varied less than 7 -fold between the tissues with the highest (flowers) and lowest (mature leaves) expression. This result is in agreement with publicly available microarray data showing expression of $P A G R$ throughout the plant (Additional file 3: Figure S3) [25].

\section{PAGR mutant alleles are not transmitted via pollen}

Two independent T-DNA insertions were identified in the coding sequence of PAGR, pagr-1 in the eleventh exon and pagr-2 in the seventh exon (Fig. 1b). We failed to isolate homozygous mutant seedlings in the progeny of plants heterozygous for either mutant allele. pagr-1 and pagr-2 mutant alleles were inherited by $41 \%$ and $44 \%$ of the progeny of heterozygous parents, indicating that the pagr mutant alleles were not transmitted by the male or female gametophyte (Table 1$) \cdot x^{2}$ tests of these segregation ratios support that both pagr mutant alleles are inherited in a 0:1:1 ratio of homozygous mutants to heterozygotes to wild type as is expected if mutant alleles are not transmitted by either the male or female gametophyte. We then performed reciprocal crosses of pagr-1 and pagr-2 heterozygotes and the wild type to test the transmission of pagr mutant alleles. In crosses where wild-type stigmas were fertilized with pollen from pagr-1 or pagr-2 heterozygotes, pagr mutant alleles were not detected in the $\mathrm{F} 1$ progeny (Table 2). When pagr-1 or pagr-2 heterozygotes were fertilized with wild-type pollen, mutant alleles were inherited by approximately $50 \%$ of the F1 progeny. $x^{2}$ tests reject the hypothesis that pagr mutant alleles were transmitted via pollen but accept the hypothesis that mutant alleles were transmitted at the expected 1:1 ratio by female gametophytes. Thus, pagr mutant alleles were not transmitted by pollen but were transmitted normally by the female gametophyte.

We therefore tested for phenotypes affecting pollen in pagr heterozygotes. The viability of pollen grains was tested with Alexander's stain in segregating wild-type and pagr heterozygous plants [26]. More than $95 \%$ of 
Table 1 Segregation ratio of PAGR mutant alleles. The genotype of offspring from selfed heterozygotic pagr mutants was determined by PCR. The observed segregation ratios are consistent with a 1:1 segregation as expected with non-functional pollen

\begin{tabular}{llllccr}
\hline Line: & pagr/pagr & pagr/PAGR & PAGR/PAGR & Proportion & p-value, $X 2$ test of 1:2:1 segregation & $p$-value, X2 test of 0:1:1 segregation \\
\hline pagr-1 & 0 & 31 & 45 & 0.41 & $7.4 \mathrm{E}-13$ & 0.11 \\
pagr-2 & 0 & 34 & 43 & 0.44 & $2.2 \mathrm{E}-11$ & 0.31 \\
\hline
\end{tabular}

pollen grains from pagr-1/PAGR, pagr-2/PAGR and $P A G R / P A G R$ plants stained purple with Alexander's stain (Fig. 2a-d) indicating that development proceeds normally in pagr pollen prior to anthesis.

Pollen from wild type, and from pagr-1/PAGR and pagr-2/PAGR heterozygotes was germinated in vitro to determine if pollen germination or pollen tube growth are affected by PAGR. We observed an approximate $50 \%$ decrease in the proportion of pollen grains producing pollen tubes in vitro from pagr-1 and pagr-2 heterozygotes (Table 3). This suggests that pagr pollen failed to germinate or to produce pollen tubes in vitro. There were no apparent alterations in the morphology of pollen tubes produced by pollen from pagr heterozygous plants (Fig. 2 e-g \& Additional file 4: Figure S4). We thus concluded that mutation of PAGR disrupts pollen germination but does not affect the development of pollen prior to germination.

\section{PAGR is localized to the Golgi apparatus and to small punctate structures}

The PAGR protein is predicted to be a type-II membrane protein (Fig. 1c) and has been detected by LC-MS/MS in the proteome of Golgi purified from Arabidopsis suspension cell cultures [27]. To confirm the localization of PAGR we transiently co-expressed PAGR-CFP in $N$. benthamiana with $\alpha$-mannosidase-1 fused to mCherry as a Golgi marker [28]. In some cells PAGR co-localized with $\alpha$-mannosidase-1 (Fig. 3a-c). However, in most cells PAGR-CFP also localized to small punctate structures in which $\alpha$-mannosidase-1 was not present (Fig. 3d-f). This localization pattern to both the Golgi apparatus and to a smaller, non-Golgi subcellular compartment is similar to the localization

Table 2 Segregation of pagr mutant alleles in the progeny of reciprocal crosses between pagr heterozygotes and wild type

\begin{tabular}{|c|c|c|c|c|}
\hline Cross & $\begin{array}{l}\text { pagr/ } \\
\text { PAGR }\end{array}$ & $\begin{array}{l}\text { PAGR/ } \\
\text { PAGR }\end{array}$ & Proportion & $\begin{array}{l}p \text {-value, } \times 2 \text { test } \\
\text { of } 1: 1 \text { segregation }\end{array}$ \\
\hline 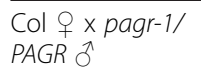 & 0 & 94 & 0.00 & $3.16 \mathrm{E}-22$ \\
\hline $\begin{array}{l}\text { pagr-1/PAGR }+x \\
\mathrm{Col} \pi\end{array}$ & 34 & 42 & 0.81 & 0.36 \\
\hline $\begin{array}{l}\text { Col } q \times \text { x pagr-2/ } \\
\text { PAGR } \sigma^{\pi}\end{array}$ & 0 & 94 & 0.00 & $3.16 \mathrm{E}-22$ \\
\hline $\begin{array}{l}\text { pagr-2/PAGR }+x \\
\text { Col } \hat{0}\end{array}$ & 47 & 46 & 1.02 & 0.92 \\
\hline
\end{tabular}

of AtGalt31A, AtGAlt29A and AtGlcAT14A [29]. AtGALT31A and AtGALT29A are galactosyltransferases involved in the biosynthesis of AGP type-II arabinogalactans [30, 31], and AtGlcAT14A is a glucuronosyltransferase also involved in the biosynthesis of type-II arabinogalactans [32]. Galt29A was reported to partially colocalize with EXO70E2 in small subcellular compartments thought to be involved in an unconventional secretion pathway known as Exocyst-Positive organelles (EXPOs) [29, 33]; however we have been unable to replicate this result.

\section{Silencing of PAGR in Nicotiana benthamiana affects pectin biosynthesis}

As we were unable to isolate homozygous Arabidopsis pagr mutants, we transiently silenced the $N$. benthamiana orthologs of PAGR, (NbPAGR) using Tobacco Rattle Virus-based Virus Induced Gene Silencing (VIGS) [34]. VIGS is a rapid and robust method for down-regulating genes of interest and has proven to be an effective system for studying plant cell wall formation and GT function $[35,36]$. We identified two PAGR orthologs in the genome of $N$. benthamiana, NbPAGR-A and NbPAGR-B [37]. NbPAGR-A and $-B$ encode proteins with $80.2 \%$ and $80.6 \%$ identity to Arabidopsis PAGR (Additional file 1: Figure S1) and phylogenetic analysis of these sequences shows strong homology to PAGR and its basal land plant orthologs (Additional file 5: Figure S5). $N b P A G R-A$ and $-B$ are $97.2 \%$ identical to one another, sufficient similarity for efficient silencing of both orthologs with a single construct. A fragment of $N b P A G R-A$ was cloned into a pTRV2 vector to induce silencing of both $N$. benthamiana PAGR orthologs. $N$. benthamiana plants were infected with TRV-VIGS viruses by infiltration with Agrobacterium containing NbPAGR and control TRV2-plasmids. NbPAGR-silenced plants had a stout phenotype resulting from cessation of internode and petiole expansion (Fig. 4). The leaves of NbPAGR silenced plants were tightly clustered around the shoot apical meristem. This phenotype is reminiscent of that resulting from silencing of UDP-Apiose/UDP-xylose synthase in $N$. benthamiana, which causes a deficiency in RG-II production [38]. We also observed alterations in root growth in $N b P A G R$-silenced plants, including decreased total root length and discoloration (Additional file 6: Figure S6). We evaluated the specificity and efficacy of gene silencing by performing real-time RT-PCR 

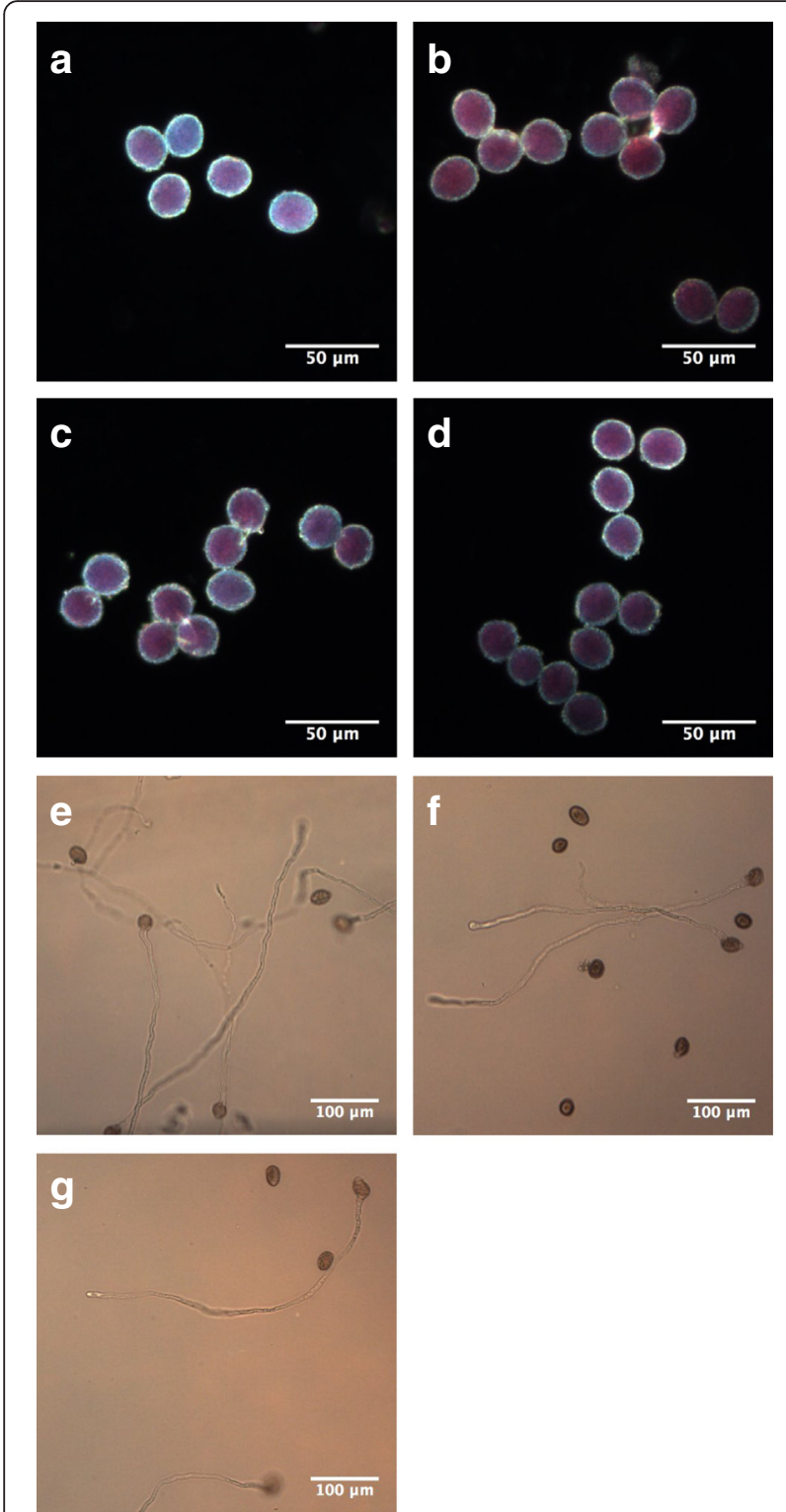

Fig. 2 Pollen phenotypes of pagr heterozygotes. Pollen from segregating PAGR/PAGR (a), pagr-1/PAGR (b), PAGR/PAGR (c) and pagr-2/PAGR (d) plants stained equally with Alexander's stain indicating that pagr pollen is viable. Scale bars $50 \mu \mathrm{m}$. In vitro pollen germination assays show reduced germination rates compared to the wild type (e) for pollen from pagr-1/PAGR (f) and pagr-2/PAGR (g) plants. Scale bars $100 \mu \mathrm{m}$

with primers specific for NbPAGR-A and $-B$. Levels of NbPAGR- $A$ and $-B$ transcripts were reduced by $34 \%$ and $26 \%$, respectively in silenced plants (Fig. 4f). While this reduction in transcript levels is relatively minor, these results were consistent, statistically significant and may be explained by cessation of growth in tissues with significant silencing.

As PAGR was predicted to be involved in the production of plant cell wall polysaccharides, we analyzed the
Table 3 Pollen tube growth analysis of pagr heterozygotes and the wild type

\begin{tabular}{llll}
\hline Pollen tube: & + & - & proportion of germinating pollen \\
\hline wild type (Col-0) & 229 & 95 & 0.71 \\
pagr-1/PAGR & 209 & 321 & $0.39^{*}$ \\
pagr-2/PAGR & 192 & 245 & $0.44^{*}$ \\
\hline
\end{tabular}

Pollen was germinated in vitro and germination rates were scored following imaging. *: $p<0.001$, population proportion Z-score test

monosaccharide composition of cell wall material from NbPAGR-silenced and virus-infected non-silenced control plants. $N b P A G R$-silenced plants exhibited a significantly altered monosaccharide composition including a $33 \%$ decrease in galactose content, a small decrease in glucuronic acid content, and increased galacturonic acid content (Fig. 4c). Sequential extraction of the cell wall material showed that less galactose and arabinose were present in pectic extracts obtained from NbPAGR-silenced cell walls with the chelating agent cyclohexane diamine tetraacetic acid (CDTA) and with sodium carbonate (Additional file 7: Figure S7). The cell wall material remaining after the extraction of pectic polysaccharides did not exhibit differences in composition between silenced and virus-infected control plants.

To determine if the observed changes in pectic galactose content were caused by a reduction in $\beta-1,4$-galactan, cell wall material was digested with endo-1,4- $\beta$-galactanase from Aspergillius niger and the solubilized and residual materials were subsequently analyzed. Treatment with the 1,4- $\beta$-galactanase released $68.9 \%$ less galactose from cell walls of NbPAGR-silenced plants than from cell walls of the control plants (Fig. 4d-e), indicating that the reduction in total galactose content was related to a significant reduction in pectic galactan content and/or enzymatic accessibility. Interestingly, less arabinose was released following $\beta-1,4$ galactanase treatment from $N b P A G R$-silenced cell wall material as well, suggesting that type-I arabinogalactans were affected by NbPAGR- silencing in addition to $\beta-1,4$-galactan. Residual material following galactanase digestion was similar in composition in NbPAGR-silenced and control cell walls (Fig. 4e).

\section{Overexpression of PAGR in Arabidopsis causes ectopic phenotypes}

To investigate the effects of PAGR overexpression we expressed PAGR-YFP in Arabidopsis under the control of the cauliflower mosaic virus $35 \mathrm{~S}$ promoter. Two independent 35S::PAGR-YFP lines were selected for further analysis. Seedlings overexpressing PAGR had a slightly dwarfed phenotype, with rosettes growing more slowly than the wild type (Fig. 5a-c). When grown under 

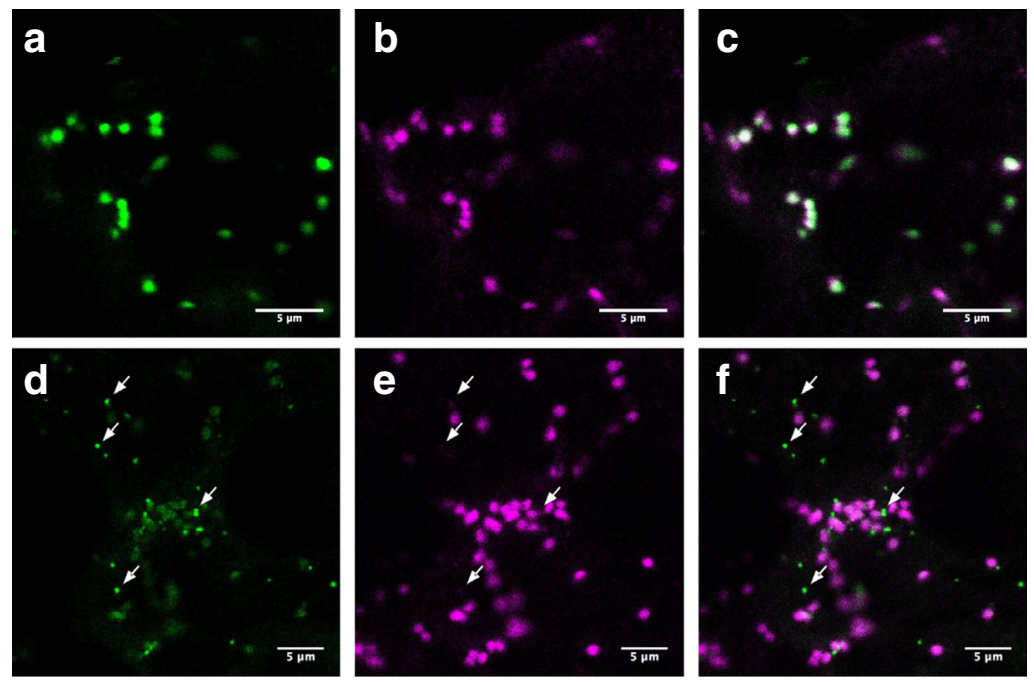

Fig. 3 The subcellular localization of PAGR-CFP. PAGR-CFP (a \& $\mathbf{d}$ ) was co-expressed with the Golgi apparatus marker a-mannosidase-1-mCherry $(\mathbf{b} \& \mathbf{e})$ in $\mathrm{N}$. benthamiana leaves; merged signals (c \& $\mathbf{f}$ ). In some cells PAGR-CFP co-localized with a-mannosidase-1-mCherry (a-c). In most cells PAGR-CFP partially co-localized with a-mannosidase-1-mCherry and also localized to small punctate structures (white arrows, $\mathbf{d}$-f). Scale bars 5 um

short-day conditions PAGR overexpressing plants lost apical dominance and developed multiple rosettes of tangled leaves (Fig. 5d-f). Under long-day conditions loss of apical dominance was observed infrequently. Inflorescences of 35S::PAGR-YFP plants had variable pleiotropic phenotypes each exhibited in independent transformants including fasciation, increased branching, swollen translucent floral pedicels and deficiencies in internode expansion (Fig. 5h-l).

Analysis of total cell wall material from three independent lines of 35S::PAGR-YFP plants showed a small increase in total arabinose content and small decreases in fucose, rhamnose and glucuronic acid content (Fig. 6a). Following sequential extraction of cell wall material from PAGR-overexpressing plants, we found that the CDTA cell wall extract contained an increased arabinose content whereas the subsequent $4 \mathrm{M} \mathrm{KOH}$ extract and the residual material showed no differences in monosaccharide composition compared to the wild type (Additional file 8: Figure S8). The expression of PAGRYFP fusion protein in transgenic plants was confirmed by immunoblotting (Fig. 6b). PAGR-YFP was detected in all transgenic lines at a molecular mass of around $110 \mathrm{kDa}$, somewhat larger than the predicted molecular mass of $92.3 \mathrm{kDa}$, with antibody against the AttB2 linker peptide present in PAGR-YFP.

NbPAGR silenced cell walls contain rhamnogalacturonan-I deficient in arabinogalactan

To further characterize the altered arabinogalactan content of cell walls from $N b P A G R$-silenced plants we analyzed the molecular mass and composition of RGI. RG-I can be solubilized from cell wall material along with RG-II by digestion with polygalacturonanase and pectin methylesterase [39]. RG-I released by this enzymatic treatment was separated from RG-II by size-exclusion chromatography on a Superdex $20010 / 300$ column (Fig. 7). RG-I from NbPAGR-silenced plants eluted later than RG-I from control plants. The average molecular mass of RG-I from silenced plants was calculated to be $91.2 \mathrm{kDa}$ compared to $118.6 \mathrm{kDa}$ in control plants when compared to the elution times of dextran molecular mass standards. RG-I fractions were collected following size exclusion chromatography and the monosaccharide composition of the collected material was analyzed. The arabinose and galactose content of RG-I was reduced from $21.9 \%$ arabinose and $50.5 \%$ galactose in control plants to $20.3 \%$ arabinose and $44.4 \%$ galactose in NbPAGR-silenced plants. Conversely the amount of RGI backbone monosaccharides rhamnose and galacturonic acid was increased from $13.3 \%$ rhamnose and $11.5 \%$ galacturonic acid in the control to $16.6 \%$ rhamnose and $15.6 \%$ galacturonic acid in silenced plants (Table 4 ).

We were then interested to determine if the decreased molecular mass of RG-I in NbPAGR-silenced plants was specifically due to reduced arabinogalactan content or if the RG-I backbone was truncated. From the approximate mass and monosaccharide composition of the isolated RG-I it is possible to estimate the approximate mass contribution of each monosaccharide comprising the RG-I molecule. First the approximate mass percentage (mass\%) of each monosaccharide for the RG-I fractions was calculated by multiplying the $\mathrm{mol} \%$ of each monosaccharide detected by its residue molecular mass 


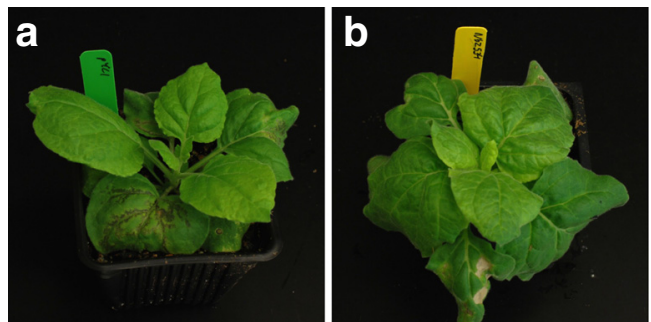

c
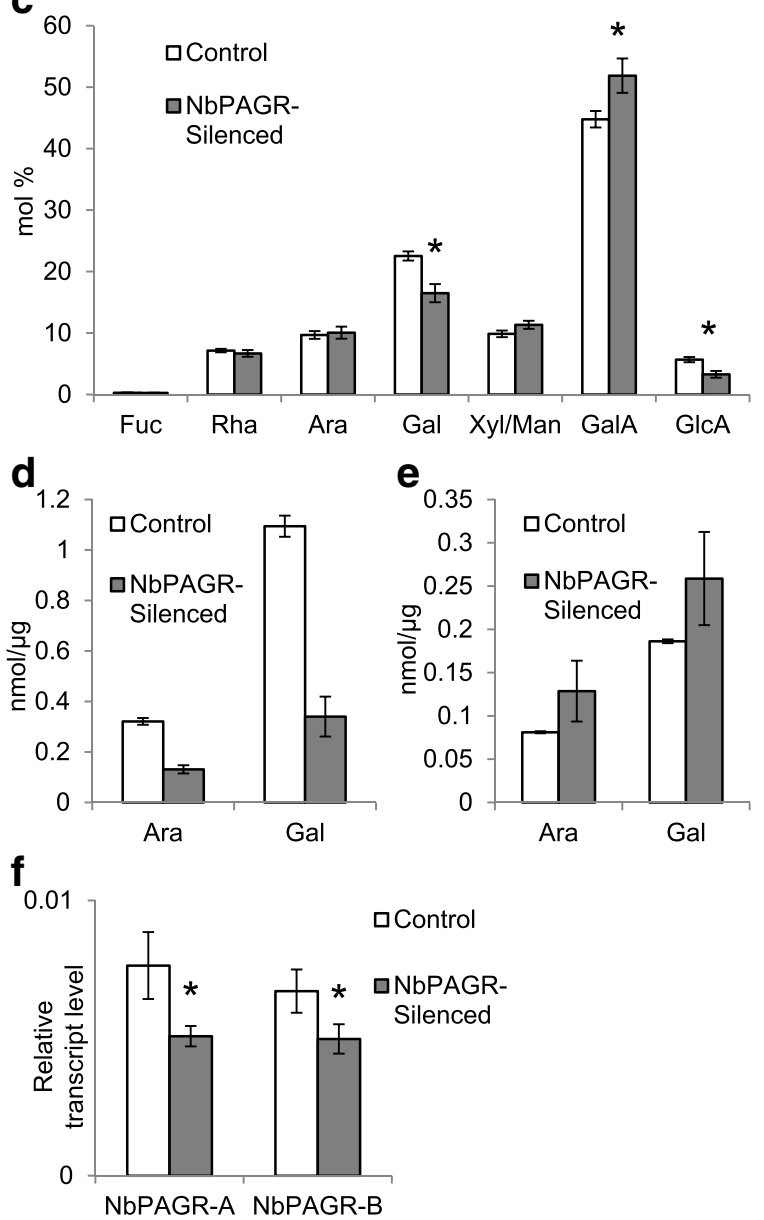

Fig. 4 The phenotype of NbPAGR-silencing in N.benthamiana. $\mathbf{a}-\mathbf{b}$ The morphological phenotype of control (a) and NbPAGRsilenced (b) plants. c The monosaccharide composition of control and NbPAGR-silenced cell walls. Values significantly different from the control are indicated with an asterisk $(n=5$, t-test, $p<0.005)$. d-e $\beta$-1,4-galactanase treatment of NbPAGR and control cell walls. Solubilized sugars (d) and residual material $(\mathbf{e})(n=3)$. f Relative expression of NbPAGR-A \& NbPAGR-B in silenced and control plants $\left(^{*}: p<0.01, n=4\right)$. Error bars indicate standard deviation

(assuming an average of one glycosidic bond per residue) and then dividing by the total of these values for each sample. The mass\% for each residue was then multiplied by the estimated total molecular mass of the RG-I fraction (Additional file 9: Table S1). This analysis showed that the amount of rhamnose and galacturonic acid residues in NbPAGR-silenced RG-I was nearly identical to that of the control with $14.9 \mathrm{kDa}$ of rhamnose and $15.5 \mathrm{kDa}$ of galacturonic acid residues in the control compared to $14.3 \mathrm{kDa}$ of rhamnose and $16.1 \mathrm{kDa}$ of galacturonic acid residues in RG-I from NbPAGR-silenced plants. The amounts of arabinose, galactose and glucuronic acid residues were decreased from $22.1 \mathrm{kDa}$ of arabinose, $62.6 \mathrm{kDa}$ of galactose and $2.6 \mathrm{kDa}$ of glucuronic acid in control plants to $15.7 \mathrm{kDa}$ of arabinose, $42.2 \mathrm{kDa}$ of galactose and $1.8 \mathrm{kDa}$ of glucuronic acid in RG-I from silenced plants. These data support that the reduced molecular mass of RG-I in NbPAGR-silenced plants is due to a reduction in arabinogalactan content of the polysaccharide, and is not due to a reduction in the degree of polymerization of the RG-I backbone. This analysis also allows us to estimate the number of residues making up each molecule of RG-I by dividing the calculated molecular mass contribution of each type of residue in the RG-I by the molecular mass of the monosaccharide residue (Table 5). This calculation yields a fairly consistent estimate of 90 to 100 rhamnose-galacturonic acid disaccharides comprising the RG-I backbone in both silenced and control plants.

\section{PAGR overexpression increases rhamnogalacturonan-I arabinan content}

RG-I from 35S::PAGR-YFP transgenic Arabidopsis seedlings was analyzed with the same method used for the NbPAGR-silenced $N$. benthamiana plants. RG-I was extracted from cell wall material enzymatically and then analyzed by size exclusion chromatography. In lines overexpressing PAGR-YFP, RG-I eluted at an earlier retention time of 20.9 and $20.8 \mathrm{~min}$ compared to $21.3 \mathrm{~min}$ in wild type seedlings (Fig. 7), corresponding to an increase in the molecular mass of RG-I from an average of 98.4 $\mathrm{kDa}$ to $106.8 \mathrm{kDa}$ and $108.3 \mathrm{kDa}$ when compared to the retention times of dextran molecular mass standards. We collected these RG-I fractions and analyzed the monosaccharide composition. In PAGR overexpressing plants we observed an increase in the mol\% of arabinose from $24.1 \%$ in the wild type to $30.2 \%$ and $33.6 \%$ in the 35S::PAGR-YFP lines (Table 6). The contribution of each monosaccharide to the total mass of the RG-I was calculated as for the RG-I from NbPAGR-silenced plants (Additional file 10: Table S2). This analysis showed an increase in the amount of arabinose residues from $20.2 \mathrm{kDa}$ in the wild type to 27.9 and $31.8 \mathrm{kDa}$ in the PAGR-overexpressing lines. There was also a decrease in the amount of glucuronic acid from $1.2 \mathrm{kDa}$ in the wild type to $1.0 \mathrm{kDa}$ and $0.8 \mathrm{kDa}$ in the overexpressor lines as well as an increase in the amount of fucose from $1.0 \mathrm{kDa}$ in the wild type to 1.3 and $1.4 \mathrm{kDa}$ in the two $P A G R$ overexpressing lines. The amount of rhamnose, galactose and galacturonic acid in RG-I from PAGR 

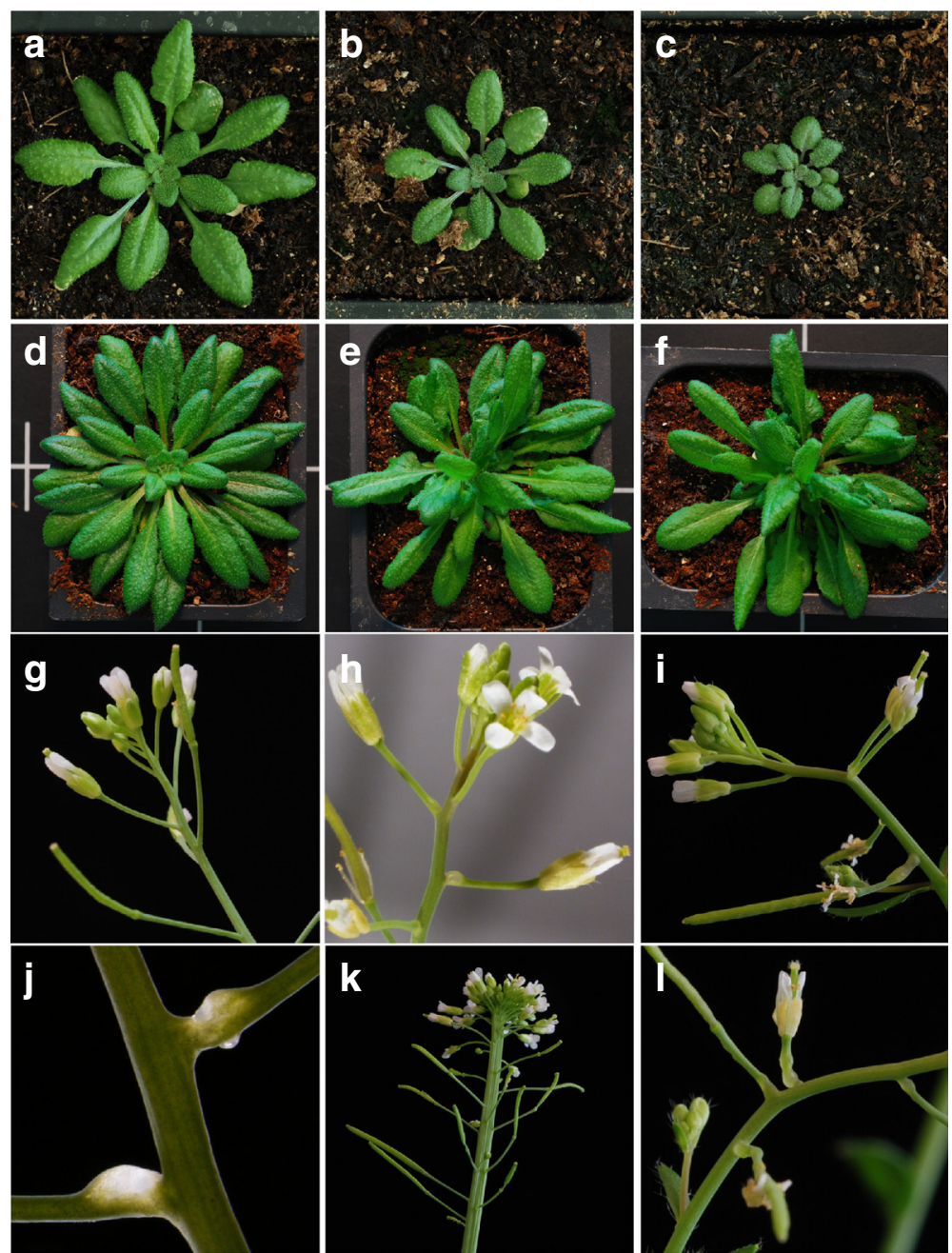

Fig. 5 PAGR overexpression phenotypes in Arabidopsis rosettes. a 4-week old wild type, b 35S::PAGR-YFP line 6, and c 35S.::PAGR-YFP line 9 plants. d 6-week old wild type, e 35S.:PAGR-YFP line 6 and $\mathbf{f}$ 35S:.:PAGR-YFP line 9 plants. 35S.:PAGR-YFP inflorescences exhibit pleiotropic phenotypes. $\mathbf{g}$ Normal wild type inflorescence. $\mathbf{h}$ Inflorescence displaying swollen floral pedicels in 35S::PAGR-YFP line 6. $\mathbf{i}$ Altered phyllotaxy and swollen pedicels in 35S.:PAGRYFP line 9. $\mathbf{j}$ Close-up of swollen pedicel tissues in 35S.:PAGR-YFP line 6. $\mathbf{k}$ Fasciation in 35S.:PAGR-YFP line 6. I Swollen pedicels and altered patterning in 35S:::PAGR-YFP line 9

overexpressors was similar to that of the wild type. Next we calculated the approximate number of each monosaccharide residue making up the RG-I fractions. Again the number of rhamnose and galacturonic acid disaccharides making up the RG-I backbone was between 90 and 100 for both overexpressing and control plants (Table 7).

\section{PAGR affects the abundance of arabinogalactan and rhamnogalacturonan-I backbone epitopes in purified rhamnogalacturonan-I}

In order to better understand the changes in RG-I composition induced by altered PAGR expression, the glycan epitope composition of RG-I samples purified by size exclusion chromatography from $N b P A G R$-silenced and control Nicotiana benthamina plants as well as
PAGR-overexpressing and wild type Arabidopsis plants were analyzed by ELISA using a comprehensive set of plant glycan-directed antibodies [40] (Fig. 7c, Additional file 11: Table S3). These RG-I preparations were recognized nearly exclusively by antibodies directed at pectic backbone and arabinogalactan epitopes. Weak binding by antibodies against de-esterified homogalacturonan (HG) epitopes was observed in RG-I preparations from all plant lines, as expected given the methods used to generate the RG-I. RG-I from NbPAGR-silenced plants yielded stronger signals for antibodies that recognize unbranched RG-I backbone epitopes than did RG-I from the controls. RG-I from silenced plants also exhibited altered signal strength for antibodies binding arabinogalactan epitopes. RG-I from silenced plants produced 


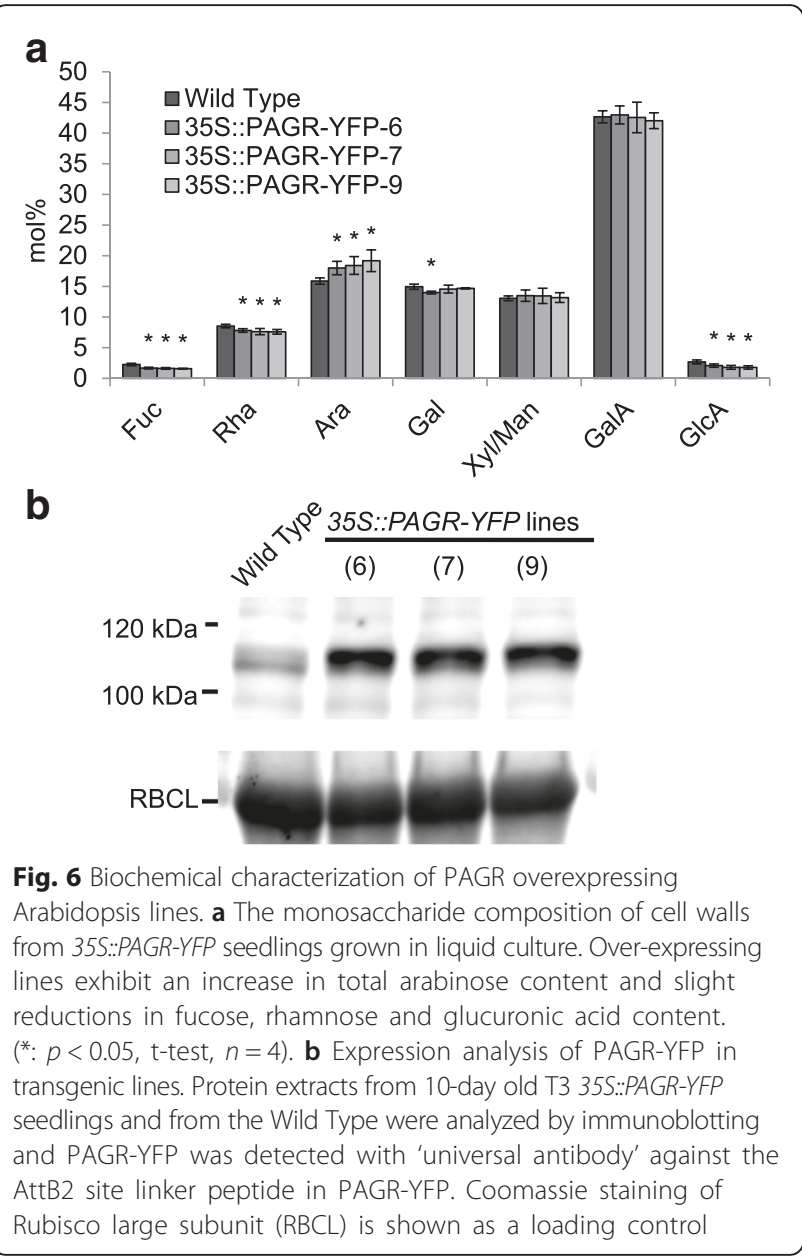

reduced signals for some antibodies belonging to the AG-2, -3 , and -4 groups of antibodies that recognize distinct arabinogalactan epitopes and subtly enhanced signals for many antibodies belonging to the AG-I group. RG-I from PAGR-overexpressing Arabidopsis plants showed no changes in signals for antibodies recognizing RG-I or HG backbone epitopes. Antibodies belonging to the AG-1 group produced stronger signals for RG-I from PAGR-overexpressing plants (lines 6 and 9) as did some antibodies in groups AG-3 and -4 that target other arabinogalactan epitopes. Thus, the ELISA analyses support the conclusion that NbPAGR-silencing affects branching of the RG-I backbone and the overall arabinogalactan composition of RG-I. ELISA results for RG-I from 35S::PAGR-YFP lines also support the conclusion that the arabinogalactan substitution of RG-I is altered in these lines.

\section{Glycosidic linkage analysis of rhamnogalacturonan-I fractions}

RG-I possesses distinct types of arabinogalactan sidechains with different galactan backbone linkages; type-I arabinogalactan has a backbone of linear $\beta-1,4-\mathrm{D}-\mathrm{Gal} p$ while type-II arabinogalactans have $\beta-1,3-, \beta-1,6$-and $\beta$ 1,3,6-linked D-Galp. To further investigate the observed changes in RG-I composition in silenced and overexpressing plants we conducted glycosidic linkage analysis by analyzing the partially methylated, partially acetylated alditol derivatives generated from purified RG-I fractions (Table 8). In RG-I from NbPAGR-silenced plants the molar percentage of 4-galactose was reduced from $47.9 \%$ in the control to $42.0 \%$. The amount of 2 rhamnose in RG-I from NbPAGR-silenced plants was increased from $6.2 \%$ in the control to $8.9 \%$. Notably the ratio of 2-rhamnose to 2,4-rhamnose was increased from $0.78: 1$ in the control to $\sim 1: 1$ in NbPAGR-silenced plants, consistent with the immunological evidence that the RG-I backbone is less branched in NbPAGR-silenced plants. Small increases in the amounts of 3,5-arabinose and 6-galactose were also detected in RG-I from NbPAGR-silenced plants. In RG-I from PAGR-overexpressing Arabidopsis plants, significant increases in the amount of 3- and 6- linked galactose were detected in addition to increases in 3,6-substituted hexose (assumed to be galactose as no glucose was detected in HPAEC glycosyl composition analysis of these fractions). The amount of 4-galactose was reduced from $19.4 \%$ in RG-I from the wild type to $16.0 \%$ and $14.7 \%$ in RG-I from the two 35S::PAGR-YFP lines. Together these data support the conclusion that the amount of type-II arabinogalactan is increased in the PAGR-overexpressing lines and the amount of type-I arabinogalactan is slightly reduced. For linkage analysis a large amount of cell wall material was required and therefore Arabidopsis rosette leaves were used as a source material, not young liquid culture-grown seedlings in which the monosaccharide composition phenotype is most pronounced in the 35S::PAGR-YFP lines. This is probably the reason that changes in RG-I arabinose content detected in RG-I purified from young seedlings were not observed in the older, soil-grown material used for linkage analysis.

\section{Discussion}

Results reported here indicate that PAGR positively affects the biosynthesis of type-II arabinogalactans when overexpressed in Arabidopsis while silencing of the $N$. benthamiana ortholog, NbPAGR, decreases substitution of RG-I with type-I arabinogalactans and reduces branching of the RG-I backbone. Proposing a simple hypothesis for the glycosyltransferase activity of PAGR to explain these discordant results is challenging. Many distinct arabinogalactan sidechain structures have been detected as branches on the RG-I backbone [41]. The pattern of these substitutions on the RG-I backbone has yet to be described. 


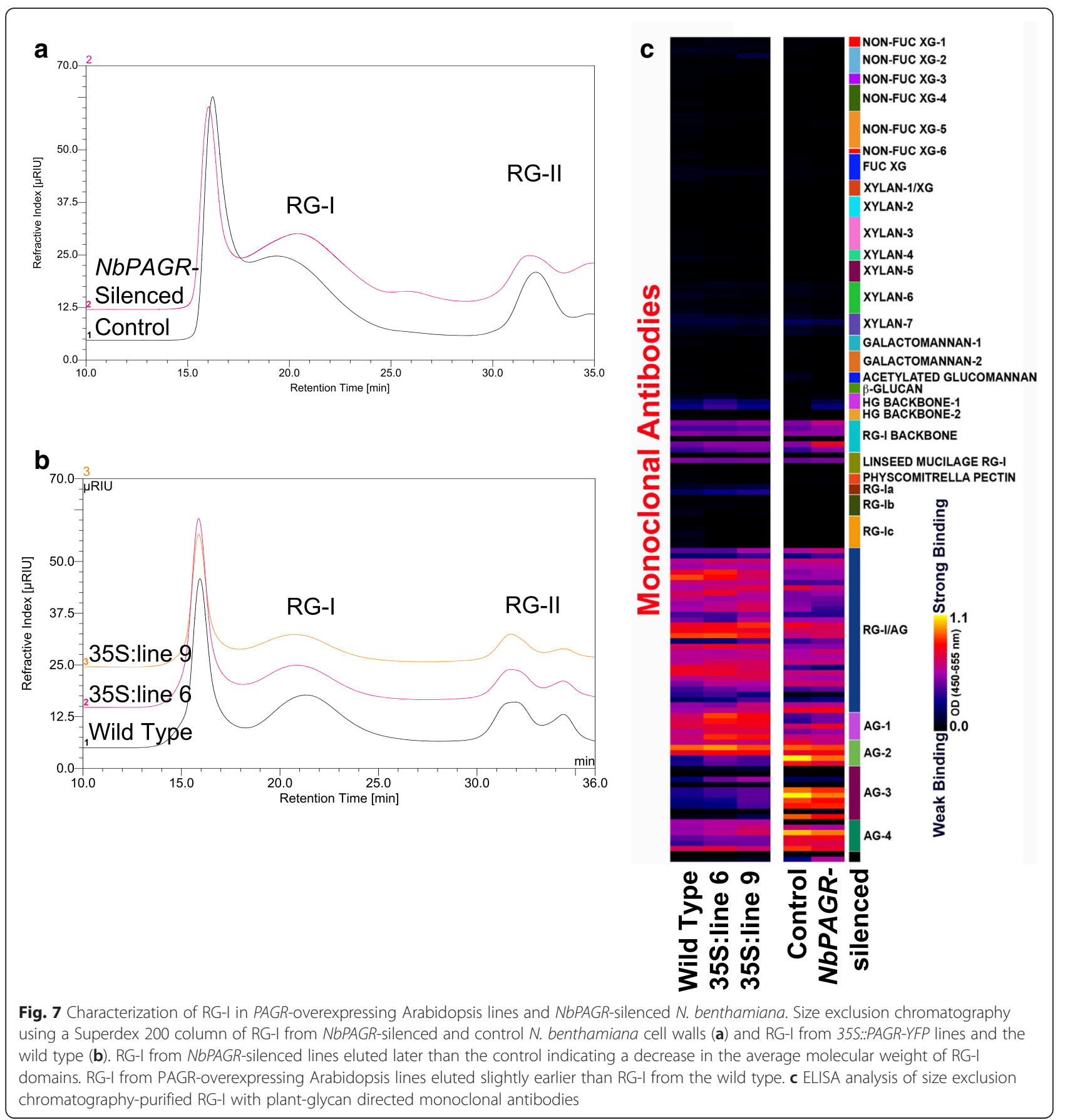

In the biosynthesis of glucuronoxylan and xyloglucan, glycosyltransferases display strong acceptor substrate specificity. In the biosynthesis of glucuronoxylan, GUX1 shows a strong preference for the addition of glucuronic acid to evenly spaced xylan residues at intervals of around 8 residues while GUX2 adds glucuronic acid at intervals of between 5 and 7 residues with no preference for even or odd spacing [42]. The activity of these two glucuronosyltransferases creates distinct xylan domains within the same molecule with distinct substitution patterns. In the biosynthesis of xyloglucan, XLT2 and MUR3 specifically add galactose to distinct xylosyl residues in each xyloglucan subunit [43, 44]. We speculate that by altering the substitution of the RG-I backbone, PAGR may affect recognition of the RG-I backbone by enzymes synthesizing type-I and type-II arabiongalactans. Alternatively, PAGR might primarily affect substitution of the most abundant RG-I arabinogalactan 
Table 4 The mol\% monosaccharide composition of RG-I purified from NbPAGR-silenced and control N. benthamiana plants

\begin{tabular}{lcc}
\hline & Control & NbPAGR-Silenced \\
\hline Rha \% & 13.3 & 16.6 \\
Ara \% & 21.9 & 20.3 \\
Gal \% & 50.5 & 44.4 \\
GalA \% & 11.5 & 15.6 \\
GlcA \% & 1.9 & 1.8 \\
\hline
\end{tabular}

sidechain. We observed that in $N$. benthamiana, where silencing of $N b P A G R$ primarily affects substitution of RG-I with type-I arabinogalactans, these are the most abundant sidechains. In Arabidopsis RG-I, type-II arabinogalactans are significantly more abundant.

The overall biosynthetic process by which pectic polysaccharides are made continues to be debated $[1,16]$. Two main models for the pectin biosynthetic process have been proposed; the consecutive GT model and the domain synthesis model. In the consecutive GT model GTs sequentially add sugars from nucleotide-sugars onto a growing pectin polysaccharide as they move through the Golgi apparatus. In the domain synthesis model oligo- or polysaccharide primers are synthesized from nucleotide sugars or lipid-linked sugars and elongated into pectic glycan domains. These pectic domains are then transferred onto a growing pectin molecule. Our data could also support PAGR being involved in a biosynthetic step prior to the transfer of glycosides or oligosaccharides onto a nascent RG-I and thus affect the production of more than one type of RG-I sidechain. This possible function for PAGR is similar to the hypothesized roles of Mannan Synthase-Related (MSR)-1 and -2 [24]. The MSRs are DUF246 domain containing, Golgi-localized GT-like proteins involved in mannan biosynthesis (see Additional file 2: Figure S2). In msr1 msr2 double mutants mannosyl levels are reduced by approximately $50 \%$ and mannan synthase activity is reduced. Wang et al., (2012) hypothesized that MSR proteins may function in the production of oligosaccharide

Table 5 The estimated average number of monosaccharide residues making up each RG-I molecule in NbPAGR-Silenced and control N. benthamiana plants

\begin{tabular}{lll}
\hline & Control & NbPAGR-Silenced \\
\hline Rha & 102 & 98 \\
Ara & 168 & 119 \\
Gal & 386 & 260 \\
GalA & 88 & 91 \\
GlcA & 15 & 10 \\
\hline
\end{tabular}

Table 6 The mol\% monosaccharide composition of RG-I purified from 35S::PAGR-YFP lines and the wild type (Col0)

\begin{tabular}{llcc}
\hline & Col-0 & 35S::PAGR-YFP line 6 & 35S-PAGR-YFP line 9 \\
\hline Fuc \% & 1.1 & 1.2 & 1.3 \\
Rha \% & 13.8 & 12.3 & 13.1 \\
Ara \% & 24.1 & 30.2 & 33.6 \\
Gal \% & 42 & 39.8 & 37.3 \\
Xyl/Man \% & 1.6 & 1.6 & 1.5 \\
GalA \% & 16.3 & 14.3 & 12.6 \\
GlcA \% & 1.1 & 0.8 & 0.6 \\
\hline
\end{tabular}

primers for the synthesis of mannans or in stabilization of the Mannan Synthase.

That PAGR appears to be necessary for pollen tube growth but not pollen development suggests that PAGR has a role in the production of polysaccharides present in the pollen tube cell wall. Experiments with transgenic expression of enzymes capable of digesting pectic polysaccharides in potatoes have shown that these polysaccharides are essential for pollen viability [21]. A collapsed pollen phenotype and decreased male fertility were observed in potato lines expressing enzymes digesting the RG-I backbone or pectic arabinans. The presence of conserved PAGR orthologs in Physcomitrella and Selaginella support that its role has been conserved throughout the evolution of land plants. The involvement of PAGR in production of a critical polysaccharide structure, such as an early bond in RG-I arabinogalactan sidechains, could drive such a high level of conservation.

The morphological phenotypes induced by overexpression and silencing of PAGR and NbPAGR provide evidence supporting a role for pectic arabinogalactans in regulating the extensibility of plant cell walls. Pleiotropic morphological phenotypes are manifested only in specific organs of PAGR overexpressors, which suggests that critical properties of RG-I are substantially altered by PAGR overexpression only in specific tissues. The

Table 7 The estimated average number of monosaccharide residues making up each RG-I molecule in 35S::PAGR-YFP and wild type (Col-0) plants

\begin{tabular}{llll}
\hline & Col-0 & 35S::PAGR-YFP line 6 & 35S-PAGR-YFP line 9 \\
\hline Fuc & 7 & 8.7 & 9.5 \\
Rha & 88 & 86 & 94 \\
Ara & 153 & 211 & 241 \\
Gal & 266 & 278 & 267 \\
Xyl/Man & 10.3 & 10.8 & 10.9 \\
GalA & 104 & 100 & 90 \\
GlcA & 6.8 & 5.4 & 4.4 \\
\hline
\end{tabular}


Table 8 Linkage analysis of RG-I from NbPAGR-silenced N. benthamiana plants and PAGR-overexpressing Arabidopsis lines

\begin{tabular}{|c|c|c|c|c|c|}
\hline & VIGS Control & NbPAGR-Silenced & Col-0 & 35S::PAGR-YFP line 6 & 35S-PAGR-YFP line 9 \\
\hline T-Ara & $1.1 \pm 0.4$ & $1.0 \pm 0.2$ & $1.9 \pm 0.5$ & $1.4 \pm 0.2$ & $2.3 \pm 0.6$ \\
\hline 2-Rha & $6.2 \pm 0.5$ & $8.9 \pm 0.1^{*}$ & $8.5 \pm 1.0$ & $7.1 \pm 1.8$ & $9.6 \pm 1.1$ \\
\hline 3-Ara & $0.7 \pm 0.1$ & $0.7 \pm 0.1$ & $1.3 \pm 0.1$ & $1.3 \pm 0.1$ & $1.2 \pm 0.1$ \\
\hline T-Gal & $4.9 \pm 0.4$ & $6.7 \pm 0.2$ & $6.3 \pm 0.3$ & $6.0 \pm 0.7$ & $5.8 \pm 0.5$ \\
\hline 5-Ara & $8.4 \pm 0.5$ & $9.2 \pm 0.7$ & $16.5 \pm 0.7$ & $16.5 \pm 0.6$ & $16.0 \pm 0.7$ \\
\hline 2,4-Rha & $7.9 \pm 0.1$ & $8.8 \pm 0.4$ & $7.4 \pm 1.2$ & $7.6 \pm 1.1$ & $6.8 \pm 0.4$ \\
\hline 3-Gal & $4.4 \pm 0.1$ & $4.5 \pm 0.3$ & $3.5 \pm 0.1$ & $4.6 \pm 0.2^{*}$ & $4.7 \pm 0.2^{*}$ \\
\hline 3,5-Ara & $1.2 \pm 0.1$ & $1.4 \pm 0.1^{*}$ & $6.4 \pm 0.2$ & $6.4 \pm 0.5$ & $5.9 \pm 0.5$ \\
\hline 4-Gal & $47.9 \pm 0.4$ & $42.0 \pm 1.6^{*}$ & $19.4 \pm 0.4$ & $16.0 \pm 0.6^{*}$ & $14.7 \pm 0.6^{*}$ \\
\hline 2,5-Ara & ND & ND & $6.6 \pm 0.4$ & $6.1 \pm 0.5$ & $5.5 \pm 0.5$ \\
\hline 6-Gal & $2.5 \pm 0.1$ & $3.0 \pm 0.2^{*}$ & $4.6 \pm 0.3$ & $5.6 \pm 0.1^{*}$ & $6.3 \pm 0.1^{*}$ \\
\hline 3,4-Hex & $1.7 \pm 0.2$ & $1.4 \pm 0.1$ & $8.9 \pm 0.5$ & $9.3 \pm 1.3$ & $8.0 \pm 1.0$ \\
\hline 2,4-Hex & $0.7 \pm 0.1$ & $0.6 \pm 0.1$ & ND & ND & ND \\
\hline 4,6-Hex & $1.6 \pm 0.1$ & $1.6 \pm 0.2$ & $1.8 \pm 0.1$ & $1.5 \pm 0.1$ & $1.3 \pm 0.1$ \\
\hline 3,6-Hex & $10.6 \pm 0.2$ & $10.4 \pm 0.4$ & $6.8 \pm 0.3$ & $10.7 \pm 0.2^{*}$ & $11.7 \pm 1.1^{*}$ \\
\hline
\end{tabular}

Values are the molar percentage of total sugars detected. Data shown are averages \pm standard deviation. Asterisks indicate significant difference from wild type or control plants $(p<0.01$, t-test). (ND: not detected)

elasticity of cell walls in the shoot apical meristem is thought to play a key role in regulating organ formation [45]. Pectin methylesterification affects the elasticity of cell walls and is highly regulated in the shoot apical meristem, where regions of de-methyl-esterification underlie new lateral organ primordia [46]. Alteration of pectin methylesterification in the shoot apical meristem also affects the elasticity of cell walls and the production of new organs [47]. The altered phyllotaxy and fasciation of 35S::PAGR-YFP plants may be attributable to altered extensibility of cell walls in the shoot apical meristem. In NbPAGR-silenced plants decreased internode expansion and shortened roots are likely due to decreased cell wall extensibility. If PAGR has a role in the biosynthesis of RG-I arabinogalactan sidechains it is possible that both the biochemical and morphological phenotypes of PAGR overexpression may depend upon the degree of RG-I substitution normally present in a particular tissue. For example, in the young PAGR-overexpressing seedlings analyzed for RG-I monosaccharide composition, we observed a significant increase in RG-I arabinan content, while the similar linkage analysis of RG-I from rosette leaves did not show alterations in arabinan in PAGRoverexpressors.

\section{Conclusions}

Together, the results presented here support that PAGR functions in the biosynthesis of RG-I arabinogalactans and illustrates the essential roles of these polysaccharides in vegetative and reproductive plant growth. More research is needed to understand the detailed structure of
RG-I, particularly with respect to the pattern and nature of branching, and the biosynthetic process by which RGI is produced in plants. Such research will better enable efforts to identify the specific biochemical role of PAGR in the biosynthesis of RG-I arabinogalactan sidechains.

\section{Methods}

\section{Plant material}

Arabidopsis thaliana Heyn. (L) accession Columbia-0 seeds were obtained from Lehle Seeds (Round Rock, Texas). Arabidopsis plants were grown under a $10-\mathrm{h}$ photoperiod $22{ }^{\circ} \mathrm{C}$ with $90 \mu \mathrm{mol} \mathrm{m}{ }^{-2} \mathrm{~s}^{-1}$ illumination during the day period. After 4 weeks, plants were transferred to a 16-h light photoperiod to induce flowering. Nicotiana benthamiana seeds were kindly provided by the Dinesh-Kumar Lab (Univerisity of California, Davis). $N$. benthamiana plants were grown under 16-h photoperiod at $25^{\circ} \mathrm{C}, 60 \%$ humidity with $200 \mu \mathrm{mol} \mathrm{m} \mathrm{m}^{-2} \mathrm{~s}^{-1}$ illumination during the day period. Arabidopsis T-DNA lines CS836448 (pagr-1) and SALK_064738C (pagr-2) were acquired from the Arabidopsis Biological Resource Center (ABRC, Ohio State University). Genotyping of T-DNA lines was performed by PCR using genomic primers Cs836448F 5'-TCTTCCAGAGATAGAGCA GATGGCTG-3' and Cs836448R 5'-TGCGCTTCTGC AAGGCGAGC-3' for pagr-1 and S_64738F 5'-TGGC GTCACTGGGTGCTCCT-3' and S_64738R 5'-TCA GCCATCTGCTCTATCTCTGGAAG-3' for $p a g r-2$. TDNAs were detected using the forward genomic primers and the appropriate left-border T-DNA primers pDAP101-Lb1 5'-GCCTTTTCAGAAATGGATAAATAG 
CCTTGCTTCC-3' for pagr-1 and LB1 5'-TGGTTCACG TAGTGGGCCATCG-3' for pagr-2. In vitro pollen germination assays were performed as described [48]. Differential staining of aborted pollen grains was performed as described [26] and imaged using a Leica DMB4000B microscope.

\section{Virus Induced Gene Silencing (VIGS)}

NbPAGR-A (Niben101Scf07590g07020) and NbPAGR-B (Niben101Scf35628g00005) were identified in the Sol Genomics Network Database [49] as reciprocal best BLAST hits for PAGR in the $N$. benthamiana genome. Alignment and phylogenetic analysis of the NbPAGR predicted amino acid sequences with the Arabidopsis DUF246 family proteins showed NbPAGR-A and -B to be the close homologs of Arabidopsis PAGR (Additional file 2: Figure S2). The NbPAGR-A sequence used to induce VIGS was amplified from total $N$. benthamiana cDNA using primers 5'-TTATCTAGACGATGACGAT TACCGTGGCCGT-3' and 5'-TTATCTAGAGCTGGTT TAGACCACCCTCAGCG-3'. Subsequently the fragment was cloned into the Xba1 site in pYL156 [34] i.e. pTRV2, to generate pYL156-NbPAGR. As a nonsilencing control plasmid, pYL156 with a fragment of the GUS gene was used (pYC1) [50]. pYC1 and pYL156NbPAGR were independently transformed into Agrobacterium tumefaciens strain GV3101. Virus-induced gene silencing was induced in 2-3 week old $N$. benthamiana plants according to standard protocols [34]. Tissue was collected 14 days post infection for all analyses. Sequence alignments were performed in Geneious 4.6.5 (Biomatters, New Zealand) Phylogenetic analyses were performed using http://www.phylogeny.fr [51].

\section{PAGR Overexpression and localization}

The coding sequence of At3g26370 (PAGR) was amplified from total Arabidopsis cDNA using Phusion HighFidelity DNA polymerase (New England Biolabs) and the primers 5'-CACCATGGCAGAGTTACGGCACTC GAGCTCTCTC-3' and 5'TCCAGCTTTACACATG CATGGAGTGAGAGG-3'. The PCR product was cloned into pENTR-D-Topo (Invitrogen). A Gateway LR recombination reaction was performed according to the manufacturer's protocol (Invitrogen) to transfer the coding sequence of $P A G R$ into pGWB41 [52] to produce 35S::PAGR-YFP for production of transgenic Arabidopsis plants. This construct was transformed into A. tumefaciens strain GV3101 and Arabidopsis plants of the Columbia-0 ecotype were transformed via the floral dip method [53]. For total cell wall and RG-I monosaccharide composition analysis of 35 :::PAGR-YFP lines, T3 seedlings were grown in liquid culture at $22{ }^{\circ} \mathrm{C}$ for 14 days. For localization studies, the coding sequence of PAGR was recombined into pGWB44 to product 35S::PAGR-CFP.
PAGR-CFP was transiently co-expressed with $\alpha$ mannosidase-1 [28] in 4-week-old $N$. benthamiana leaves following described procedures [54] except that $100 \mathrm{mM}$ 2-(N-morpholino)ethanesulfonic acid, $100 \mathrm{mM}$ $\mathrm{MgCl}_{2}, 10 \mu \mathrm{M}$ acetosyringone was used as the infiltration medium. Expression in N. benthamiana epidermal cells was imaged using a Zeiss 710 confocal laser-scanning microscope (Carl Zeiss).

\section{Immunoblotting}

Total protein was extracted from 1 week old Arabidopsis seedlings by grinding in $100 \mathrm{mM}$ Tris $\mathrm{pH} 7.5,1 \mathrm{mM}$ EDTA, $1 \%$ (v/v) Triton X-100, $10 \%$ (v/v) glycerol, $1 \mathrm{mM}$ phenylmethylsulfonyl fluoride. Cellular debris was pelleted by centrifugation at $16,000 \times \mathrm{g}$ for $15 \mathrm{~min}$ at $4{ }^{\circ} \mathrm{C}$. Extracted protein was quantified by Bradford Assay [55]. For electrophoresis, $40 \mu \mathrm{g}$ of protein was separated by SDS-PAGE, electrotransferred onto a PVDF membrane and incubated with AttB2 site 'universal' primary antibody and anti-rabbit secondary antibody as described by [56], except that $5 \% \mathrm{w} / \mathrm{v}$ BSA (Sigma) in TBS$\mathrm{T}$ was used as a blocking agent and membranes were incubated with universal antibody at a 1:3000 dilution.

\section{Quantitative RT-PCR}

RNA was extracted from Arabidopsis and N. benthamiana tissues using the RNEasy plant mini kit (Qiagen). For cloning purposes RNA was extracted from $N$. benthamiana tissues using Trizol Reagent (Invitrogen). cDNA was synthesized with Superscript-III reverse transcriptase (Invitrogen). Quantitative RT-PCR was performed on a StepOne-Plus Real-Time PCR system (Applied BioSystems) using Syber-Select Real-Time PCR reagents (Invitrogen). PAGR was detected using primer At3g26370 q1F 5'-GAGGTCGTCGCAGATCTT CAGGTTCATGT-3' and At3g26370 q1R 5'-GGCTCC CACTGTTCTTCTTCATCAGGCTT-3'. MONENSIN SENSITIVITY1, At2g28390, a gene with exceptional transcript-level stability [57], was analyzed as a reference gene using primers 5'AACTCTATGCAGCA TTTGATCCACT-3' and 5'-TGATTGCATATCTTTAT CGCCATC-3'. Data were analyzed using the comparative $\mathrm{Ct}$ method. For quantitative real-time RT-PCR analysis of NbPAGR silencing, data were analyzed using the geometric mean of three reference genes as the common reference as described [58]. For NbPAGR-A and $-B$, primers NbPAGR-AqF 5'-CTACGCCACTCAAGCTCGATCGGA AA-3', NbPAGR-AqR 5'-GCCACGGTAATCGTCATCGT CATCGTCAA-3', NbPAGR-BqF 5'-CTACGCCACTCAA GCTCGATCGGAAG-3' and NbPAGR-BqR 5'-GCCACG GTAATCGTCATCGTCATCGTCAT-3' were used. Elongation Factor 1A, Actin-2 and Ubiquitin 3 were used as reference genes using Elongation Factor $1 \mathrm{~A}$ primers NbEF1qF 5'-AGGGTCCAACCCTCCTTGAGGC-3' and 
NbEF1qR 5'-GCCCCTTTGGCTGGGTCGTC-3'; Actin-2 primers ACT2F 5'-TTGAGACTTTTAATACCCCAGC-3' and ACT2R 5'-AACATGTAACCACGCTCGGTAA-3' and Ubiquitin-3 primers UBQ3F 5'-GCCGATTACAA CATCCAGAAGG-3' and UBQ3R 5'-TGAAGTACAGC GAGCTTAACC-3'.

\section{Cell wall isolation and monosaccharide composition analysis}

Alcohol-insoluble residue (AIR) was prepared as described by [12]. Lyophilized AIR was hydrolyzed in $2 \mathrm{M}$ trifluoroacetic acid at $120{ }^{\circ} \mathrm{C}$ for $1 \mathrm{~h}$ and analyzed by high-performance anion exchange chromatography (HPAEC) as described by [59]. Glucose was not determined for samples of total cell wall material due to the presence of residual starch. Sequential extraction of AIR was performed essentially as described [60] with the exception that samples from PAGR-overexpressors were not extracted with $1 \mathrm{M} \mathrm{KOH}$ prior to the extraction with $4 \mathrm{M} \mathrm{KOH}$.

\section{Endo- $\beta$-1,4-galactanase digestion}

Cell wall preparations from VIGS plants were further analyzed by digestion with endo- $\beta$-1,4-galactanase from Aspergillus niger purified to a single band on a silverstained gel (Megazyme, product code E-EGALN). AIR $(2 \mathrm{mg}$ ) was dissolved in $0.1 \mathrm{~mL}$ of $1 \mathrm{M} \mathrm{KOH}$ and adjusted to $\mathrm{pH} 4.7$ with $2 \mathrm{~mL}$ of $100 \mathrm{mM}$ acetic acid. Galactanase was added and samples were incubated at $40{ }^{\circ} \mathrm{C}$ for $1 \mathrm{~h}$ at $40{ }^{\circ} \mathrm{C}$. After incubation, cold ethanol with $10 \mathrm{mM}$ EDTA was added to a final concentration of $70 \%(\mathrm{v} / \mathrm{v})$ and the sample was centrifuged for $5 \mathrm{~min}$ at $14,000 \times \mathrm{g}$ at $4{ }^{\circ} \mathrm{C}$. The supernatant and pellet were separated, hydrolyzed and analyzed by HPAEC as described above.

\section{Rhamnogalacturonan-I purification}

Rhamnogalacturonan-I was isolated essentially as described by [39]. Briefly, $15 \mathrm{mg}$ of AIR was digested overnight with $3 \mathrm{U}$ of pectin methyl-esterase (Novoshape Pure PME, Novozymes) and 20U of endopolygalacturonanase M2 (Megazyme, product code E-PGALUSP) at $37{ }^{\circ} \mathrm{C}$ in $1 \mathrm{ml}$ of $50 \mathrm{mM}$ ammonium oxalate ( $\mathrm{pH} \mathrm{5.0)}$. Following digestion, insoluble material was removed by centrifugation followed by filtration through a $0.2 \mu \mathrm{m}$ spin filter. Oligosaccharides and the digestion buffer were removed by washing of the solubilized polysaccharides on a $10 \mathrm{kDa}$ Molecular Weight Cutoff spin filter (Amicon) with sterile water. Samples were eluted from the spin concentrators in water and separated by size-exclusion chromatography in $50 \mathrm{mM}$ ammonium formate (pH5.0) on a Superdex 200 10/300GL column (GE Healthcare BioSciences, http://www.gelifesciences.com/) at a flow rate of $0.5 \mathrm{ml} / \mathrm{min}$. Elution of polysaccharides from the column was monitored with a Shodex RI-101 refractive index detector (Shodex, http://www.shodex.com). Fractions were collected manually, lyophilized, hydrolyzed and analyzed by HPAEC as described above. Estimates of the MW of RG-I were made with reference to the retention times of Dextran MW standards (Sigma-Aldrich). The relative mass percentage of each monosaccharide in the RG-I fractions was determined by first calculating the mass ratio of each monosaccharide by dividing the product of the mol\% and the molar mass of each monosaccharide by the sum of the products of the molar mass and mol\% of each monosaccharide. We then multiplied the monosaccharide mass ratios by the estimated molecular weight of the RG-I fraction.

\section{ELISA screening of purified rhamnogalacturonan-I preparations}

Purified RG-I samples were dissolved in water and were coated onto ELISA plates [384 well clear flat bottom polystyrene high bind microplate (product no. 3700), Corning Life Sciences] on an equal weight (gravimetric) per well basis $(0.5 \mu \mathrm{g} /$ well $)$. The samples were then subjected to ELISA screening with a comprehensive suite of cell wall glycan-directed monoclonal antibodies essentially as described earlier [40, 61]. The ELISA screening assays were done using an Robotic System (Thermo Scientific) comprising an Orbitor RS Robotic Arm (Thermo Scientific) accessing the following components: Carousel plate storage/incubation (Thermo Scientific), EON Plate Reader (Biotek), EL406 ELISA Plate Washer (Biotek), Multiflo Dispenser (Biotek) and Precision XS Fluid Dispenser (Biotek). The whole system is operated by the laboratory automation software, Momentum 3.2.7 (Thermo Scientific). Water was used as the blanks and these background values were subtracted from the sample ELISA responses. The ELISA assays were done in technical duplicates and data represent the average of the replicates. Cell wall glycan-directed antibodies were were obtained from laboratory stocks (CCRC, JIM and MAC series) at the Complex Carbohydrate Research Center (available through CarboSource Services; http:// www.carbosource.net) or through BioSupplies (Australia) (BG1, LAMP).

\section{Rhamnogalacturonan-I linkage analysis}

Glycosidic linkage analysis of RG-I was performed by GC/MS analysis of their partially methylated alditol acetates [62]. Purified RG-I samples were per-O-methylated using liquid $\mathrm{NaOH}$ in dimethyl sulfoxide and further derivatized to their corresponding partially methylated alditol acetates by trifluoroacetic acid hydrolysis, reduction and per-O-acetylation. The derivatives were separated using a gas chromatograph (Agilent 7890A, Agilent Technologies, www.agilent.com) equipped with a Supelco SP2380 column (Sigma-Aldrich) 
and a mass spectrometer (Agilent 5975C) using a temperature gradient as described [60]. Eluted compounds were identified based on their retention time compared to standards and their ion fragmentation patterns.

\section{Ethics approval and consent to participate \\ Not applicable.}

\section{Consent for publication}

Not applicable.

\section{Availability of data and materials}

The data sets supporting the results of this article are included within the article and its additional files. Nucleotide sequences and biological materials including Arabidopsis seeds, plasmids and bacterial strains created through this work are available at (https:// registry.jbei.org). Data supporting phylogenetic analyses presented in this study are available at (http:// purl.org/phylo/treebase/phylows/study/TB2:S19106).

\section{Additional files}

Additional file 1: Figure S1. Multiple amino acid sequence alignment of the Arabidopsis thaliana, Nicotiana benthamiana, Selaginella moellendorfii and Physcomitrella patens orthologs of PAGR. * identical amino acids, : conserved substitutions, . semi-conserved substitutions. (PPTX $36 \mathrm{~kb}$ )

Additional file 2: Figure S2. Phylogenetic analysis of the DUF246 containing proteins in Arabidopsis, Selaginella moellendorffi and Physcomitrella patens. After manual curation of sequences, phylogenetic analysis was performed using MUSCLE alignment, phyML and statistical analysis using $\mathrm{SH}$-like approximate likelihood-test. The tree was generated using http://Www.phylogeny.fr/ [49]. (PNG $187 \mathrm{~kb}$ )

Additional file 3: Figure S3. Tissue specific microarray data heat map depicting the relative expression level of PAGR in various tissues throughout growth and development. Publicly available data was retrieved and displayed using the Arabidopsis eFP browser (http:// bar.utoronto.ca/efp/cgi-bin/efpWeb.cgi) [23]. (PPTX 235 kb)

Additional file 4: Figure S4. Root phenotypes of NbPAGR-silenced and TRV-infected unsilenced control plants. (A) Roots of NbPAGR-silenced plants are shorter than those of control plants and were discolored. (B) Roots of NbPAGR-silenced and control plants were measured and were found to be significantly shorter in $s^{*} p<0.05, n=4$, brackets indicate one standard deviation (B). Discoloration of NbPAGR-silenced roots (D) compared to control roots (C). (PPTX $4221 \mathrm{~kb}$ )

Additional file 5: Figure S5. Additional images of in vitro pollen germination assays showing reduced germination rates in pollen from pagr-1 and pagr-2 heterozygous plants compared to the wild type. Scale bars are $100 \mu \mathrm{m}$. (PPTX $1073 \mathrm{~kb}$ )

Additional file 6: Figure S6. Phylogenetic analysis of the DUF246 containing proteins in Arabidopsis and the PAGR orthologs identified in Nicotiana benthamiana, Selaginella moellendorffi and Physcomitrella patens. After manual curation of sequences, phylogenetic analysis was performed using MUSCLE alignment, phyML and statistical analysis using SH-like approximate likelihood-test. The tree was generated using http:// www.phylogeny.fr/ [49]. (PNG 23 kb)

Additional file 7: Figure S7. Sequential extraction of cell wall material from NbPAGR-silenced Nicotiana benthamiana plants. Cell wall material from NbPAGR-silenced and control plants was sequentially extracted with CDTA, sodium carbonate, $1 \mathrm{M} \mathrm{KOH}$ and $4 \mathrm{M} \mathrm{KOH}$. The monosaccharide composition of the extracted and residual materials was then analyzed. Cell wall polysaccharides with reduced galactan and increased glucuronic acid content in PAGR-silenced plants were extracted with the CDTA and sodium carbonate fractions. ${ }^{*}: p<0.001$, t-test, $n=4$. (PPTX $47 \mathrm{~kb}$ )

Additional file 8: Figure S8. Sequential extraction of cell walls from 35S:PAGR-YFP expressing Arabidopsis seedlings. Cell wall material from was sequentially extracted with CDTA, sodium carbonate, and $4 \mathrm{M} \mathrm{KOH}$. The monosaccharide composition of the extracted and residual materials was then analyzed. Cell wall polysaccharides with increased arabinan content were extracted in the CDTA fraction. *: $p<0.001$, t-test, $n=4$. (PPTX $45 \mathrm{~kb}$ )

Additional file 9: Table S1. The approximate mass ( $k D a)$ of each monosaccharide residue in RG-I from NbPAGR-silenced and control N. benthamiana plants. (DOCX 39 kb)

Additional file 10: Table S2. The approximate mass $(\mathrm{kDa})$ of each monosaccharide residue in RG-I purified from 35S.:PAGR-YFP and wild type (Col-0) plants. (DOCX 44 kb)

Additional file 11: Table S3. ELISA absorbance values for RG-I fractions from NbPAGR-silenced and virus-infected, control N. benthamiana plants as well as 35S.:.PAGR-YFP and wild type Arabidopsis plants probed with a diverse array of plant cell wall glycan-directed monoclonal antibodies. (XLSX $21 \mathrm{~kb})$

\section{Competing interests}

The authors declare that they have no competing interests.

\section{Authors' contributions}

SS contributed to the experimental design, performed the experiments and wrote a first draft of the manuscript. BE, GX, SP, DB, JL, and MGH performed experiments and contributed to the analysis of the data. MP and JLH contributed to the experimental design and writing of the manuscript. HVS and SS conceived the study, contributed to the experimental design, coordinated the experiments, and prepared the last version of the manuscript. All authors read and approved the final manuscript.

\section{Funding}

This work was supported by the U. S. Department of Energy, Office of Science, Office of Biological and Environmental Research, through contract DE-AC02-05CH11231 between Lawrence Berkeley National Laboratory and the U. S. Department of Energy. Part of the work was supported by the Danish Strategic Research Council (Set4Future 11-116795). The generation of the CCRC series of plant cell wall glycan-directed monoclonal antibodies used for ELISA screening in this work was supported by the United States National Science Foundation Plant Genome Program (DBI-0421683 and IOS-0923992).

\section{Author details}

1Joint BioEnergy Institute and Biological Systems and Engineering, Lawrence Berkeley National Laboratory, Berkeley, CA 94720, USA. ²Department of Plant and Environmental Sciences, Faculty of Science, University of Copenhagen, C 1871 Copenhagen, Denmark. ${ }^{3}$ Energy Biosciences Institute, University of California, Berkeley, CA 94720, USA. ${ }^{4}$ Department of Plant and Microbial Biology, University of California, Berkeley, CA 94720, USA. ${ }^{5}$ Complex Carbohydrate Research Center, University of Georgia, Athens, GA 30602-4712, USA. ${ }^{6}$ BioEnergy Science Center, University of Georgia, Athens, GA 30602-4712, USA. ${ }^{7}$ Department of Plant Biology, University of Georgia, Athens, GA 30602-4712, USA. ${ }^{8}$ ARC Centre of Excellence in Plant Cell Walls, School of Botany, The University of Melbourne, 3010 Melbourne, Victoria, Australia.

Received: 12 January 2016 Accepted: 13 April 2016

Published online: 18 April 2016

\section{References}

1. Atmodjo MA, Hao Z, Mohnen D. Evolving views of pectin biosynthesis. Annu Rev Plant Biol. 2013;64:747.

2. Keegstra K, Talmadge KW, Bauer WD, Albersheim P. The Structure of Plant Cell Walls: III. A Model of the Walls of Suspension-cultured Sycamore Cells 
Based on the Interconnections of the Macromolecular Components. Plant Physiol. 1973:51:188.

3. Lau JM, McNeil M, Darvill AG, Albersheim P. Structure of the backbone of rhamnogalacturonan I, a pectic polysaccharide in the primary cell walls of plants. Carbohydr Res. 1985;137:111.

4. Bar-Peled M, Urbanowicz BR, O'Neill MA. The Synthesis and Origin of the Pectic Polysaccharide Rhamnogalacturonan II - Insights from Nucleotide Sugar Formation and Diversity. Front Plant Sci. 2012;3:92.

5. O'Neill MA, Ishii T, Albersheim P, Darvill AG. Rhamnogalacturonan II: structure and function of a borate cross-linked cell wall pectic polysaccharide. Annu Rev Plant Biol. 2004;55:109.

6. Breton C, Snajdrová L, Jeanneau C, Koca J, Imberty A. Structures and mechanisms of glycosyltransferases. Glycobiology. 2006;16:29R.

7. Lombard V, Golaconda Ramulu H, Drula E, Coutinho PM, Henrissat B. The carbohydrate-active enzymes database (CAZy) in 2013. Nucleic Acids Res. 2014;42:D490

8. Sterling JD, Atmodjo MA, Inwood SE, Kolli VSK, Quigley HF, Hahn MG Mohnen D. Functional identification of an Arabidopsis pectin biosynthetic homogalacturonan galacturonosyltransferase. Proc Natl Acad Sci U S A. 2006;103:5236

9. Kong Y, Zhou G, Abdeen AA, Schafhauser J, Richardson B, Atmodjo MA, Jung J, Wicker L, Mohnen D, Western T, Hahn MG. GALACTURONOSYLTRANSFERASELIKE5 is involved in the production of Arabidopsis seed coat mucilage. Plant Physiol. 2013;163:1203.

10. Egelund J, Petersen BL, Motawia MS, Damager I, Faik A, Olsen CE, Ishii T, Clausen H, Ulvskov P, Geshi N. Arabidopsis thaliana RGXT1 and RGXT2 encode Golgi-localized (1,3)-alpha-D-xylosyltransferases involved in the synthesis of pectic rhamnogalacturonan-II. Plant Cell. 2006;18:2593.

11. Egelund J, Damager I, Faber K, Olsen CE, Ulvskov P, Petersen BL. Functional characterisation of a putative rhamnogalacturonan II specific xylosyltransferase. FEBS Lett. 2008:582:3217.

12. Harholt J, Jensen JK, Sorensen SO, Orfila C, Pauly M, Scheller HV. ARABINAN DEFICIENT 1 is a putative arabinosyltransferase involved in biosynthesis of pectic arabinan in Arabidopsis. Plant Physiol. 2006;140:49.

13. Harholt J, Jensen JK, Verhertbruggen Y, Sogaard C, Bernard S, Nafisi M, Poulsen CP, Geshi N, Sakuragi Y, Driouich A, Knox JP, Scheller HV. ARAD proteins associated with pectic Arabinan biosynthesis form complexes when transiently overexpressed in planta. Planta. 2012;236:115.

14. Jensen JK, Sorensen SO, Harholt J, Geshi N, Sakuragi Y, Moller I, Zandleven J, Bernal AJ, Jensen NB, Sorensen C, Pauly M, Beldman G, Willats WG, Scheller HV. Identification of a xylogalacturonan xylosyltransferase involved in pectin biosynthesis in Arabidopsis. Plant Cell. 2008;20:1289.

15. Liwanag AJ, Ebert B, Verhertbruggen Y, Rennie EA, Rautengarten C, Oikawa A, Andersen MC, Clausen MH, Scheller HV. Pectin biosynthesis: GALS1 in Arabidopsis thaliana is a beta-1,4-galactan beta-1,4-galactosyltransferase. Plant Cell. 2012:24:5024.

16. Tan L, Eberhard S, Pattathil S, Warder C, Glushka J, Yuan C, Hao Z, Zhu X, Avci U, Miller JS, Baldwin D, Pham C, Orlando R, Darvill A, Hahn MG, Kieliszewski MJ, Mohnen D. An Arabidopsis cell wall proteoglycan consists of pectin and arabinoxylan covalently linked to an arabinogalactan protein. Plant Cell. 2013;25:270.

17. Popper ZA, Michel G, Herve C, Domozych DS, Willats WG, Tuohy MG Kloareg B, Stengel DB. Evolution and diversity of plant cell walls: from algae to flowering plants. Annu Rev Plant Biol. 2011;62:567.

18. Domozych DS, Sorensen I, Popper ZA, Ochs J, Andreas A, Fangel JU, Pielach A, Sacks C, Brechka H, Ruisi-Besares P, Willats WG, Rose JK. Pectin Metabolism and Assembly in the Cell Wall of the Charophyte Green Alga Penium margaritaceum. Plant Physiol. 2014;165:105.

19. Rounds CM, Bezanilla M. Growth mechanisms in tip-growing plant cells. Annu Rev Plant Biol. 2013;64:243.

20. Castro AJ, Suarez C, Zienkiewicz K, Alche Jde D, Zienkiewicz A, Rodriguez-Garcia MI. Electrophoretic profiling and immunocytochemical detection of pectins and arabinogalactan proteins in olive pollen during germination and pollen tube growth. Ann Bot. 2013;112:503.

21. Cankar K, Kortstee A, Toonen MA, Wolters-Arts M, Houbein R, Mariani C, Ulvskov P, Jorgensen B, Schols HA, Visser RG, Trindade LM. Pectic arabinan side chains are essential for pollen cell wall integrity during pollen development. Plant Biotechnol J. 2014;12:492.

22. Hansen SF, Harholt J, Oikawa A, Scheller HV. Plant Glycosyltransferases Beyond CAZy: A Perspective on DUF Families. Front Plant Sci. 2012;3:59.
23. Wang Y, Shao L, Shi S, Harris RJ, Spellman MW, Stanley P, Haltiwanger RS. Modification of epidermal growth factor-like repeats with O-fucose. Molecular cloning and expression of a novel GDP-fucose protein Ofucosyltransferase. J Biol Chem. 2001:276:40338.

24. Wang Y, Mortimer JC, Davis J, Dupree P, Keegstra K. Identification of an additional protein involved in mannan biosynthesis. Plant J. 2013;73:105.

25. Winter D, Vinegar B, Nahal H, Ammar R, Wilson GV, Provart NJ. An "Electronic Fluorescent Pictograph" browser for exploring and analyzing large-scale biological data sets. PLoS One. 2007;2:e718,

26. Peterson R, Slovin JP, Chen C. A simplified method for differential staining of aborted and non-aborted pollen grains. Int J Plant Biol. 2010;1:13.

27. Parsons HT, Christiansen K, Knierim B, Carroll A, Ito J, Batth TS, Smith-Moritz AM, Morrison S, Mclnerney P, Hadi MZ, Auer M, Mukhopadhyay A, Petzold CJ, Scheller HV, Loque D, Heazlewood JL. Isolation and proteomic characterization of the Arabidopsis Golgi defines functional and novel components involved in plant cell wall biosynthesis. Plant Physiol. 2012;159:12.

28. Nelson BK, Cai X, Nebenfuhr A. A multicolored set of in vivo organelle markers for co-localization studies in Arabidopsis and other plants. Plant J. 2007;51:1126.

29. Poulsen CP, Dilokpimol A, Mouille G, Burow M, Geshi N. Arabinogalactan glycosyltransferases target to a unique subcellular compartment that may function in unconventional secretion in plants. Traffic. 2014;15:1219.

30. Geshi N, Johansen JN, Dilokpimol A, Rolland A, Belcram K, Verger S, Kotake T, Tsumuraya Y, Kaneko S, Tryfona T, Dupree P, Scheller HV, Hofte H, Mouille G. A galactosyltransferase acting on arabinogalactan protein glycans is essential for embryo development in Arabidopsis. Plant J. 2013;76:128.

31. Dilokpimol A, Poulsen CP, Vereb G, Kaneko S, Schulz A, Geshi N. Galactosyltransferases from Arabidopsis thaliana in the biosynthesis of type I arabinogalactan: molecular interaction enhances enzyme activity. BMC Plant Biol. 2014;14:90

32. Knoch E, Dilokpimol A, Tryfona T, Poulsen CP, Xiong G, Harholt J, Petersen BL, Ulvskov P, Hadi MZ, Kotake T, Tsumuraya Y, Pauly M, Dupree P, Geshi N. A beta-glucuronosyltransferase from Arabidopsis thaliana involved in biosynthesis of type II arabinogalactan has a role in cell elongation during seedling growth. Plant J. 2013;76:1016.

33. Wang J, Ding Y, Wang J, Hillmer S, Miao Y, Lo SW, Wang X, Robinson DG, Jiang L. EXPO, an exocyst-positive organelle distinct from multivesicular endosomes and autophagosomes, mediates cytosol to cell wall exocytosis in Arabidopsis and tobacco cells. Plant Cell. 2010;22:4009.

34. Liu Y, Schiff M, Marathe R, Dinesh Kumar SP. Tobacco Rar1, EDS1 and NPR1/ $\mathrm{NIM} 1$ like genes are required for $\mathrm{N}$-mediated resistance to tobacco mosaic virus. Plant J. 2002;30:415.

35. Zhu X, Pattathil S, Mazumder K, Brehm A, Hahn MG, Dinesh-Kumar SP, Joshi CP. Virus-induced gene silencing offers a functional genomics platform for studying plant cell wall formation. Mol Plant. 2010;3:818.

36. Rennie EA, Ebert B, Miles GP, Cahoon RE, Christiansen KM, Stonebloom S, Khatab H, Twell D, Petzold CJ, Adams PD, Dupree P, Heazlewood JL, Cahoon EB, Scheller HV. Identification of a sphingolipid alphaglucuronosyltransferase that is essential for pollen function in Arabidopsis. Plant Cell. 2014;26:3314

37. Bombarely A, Rosli HG, Vrebalov J, Moffett P, Mueller LA, Martin GB. A draft genome sequence of Nicotiana benthamiana to enhance molecular plantmicrobe biology research. Mol Plant Microbe Interact. 2012;25:1523.

38. Ahn JW, Verma R, Kim M, Lee JY, Kim YK, Bang JW, Reiter WD, Pai HS Depletion of UDP-D-apiose/UDP-D-xylose synthases results in rhamnogalacturonan-II deficiency, cell wall thickening, and cell death in higher plants. J Biol Chem. 2006;281:13708.

39. Egelund J, Obel N, Ulvskov P, Geshi N, Pauly M, Bacic A, Petersen BL. Molecular characterization of two Arabidopsis thaliana glycosyltransferase mutants, rral and rra2, which have a reduced residual arabinose content in a polymer tightly associated with the cellulosic wall residue. Plant Mol Biol. 2007;64:439.

40. Pattathil S, Avci U, Baldwin D, Swennes AG, McGill JA, Popper Z, Bootten T, Albert A, Davis RH, Chennareddy C, Dong R, O'Shea B, Rossi R, Leoff C, Freshour G, Narra R, O'Neil M, York WS, Hahn MG. A comprehensive toolkit of plant cell wall glycan-directed monoclonal antibodies. Plant Physiol. 2010;153:514.

41. Lau JM, McNeil M, Darvill AG, Albersheim P. Treatment of rhamnogalacturonan I with lithium in ethylenediamine. Carbohydr Res. 1987;168:245.

42. Bromley JR, Busse-Wicher M, Tryfona T, Mortimer JC, Zhang Z, Brown DM, Dupree P. GUX1 and GUX2 glucuronyltransferases decorate distinct domains of glucuronoxylan with different substitution patterns. Plant J. 2013;74:423. 
43. Madson M, Dunand C, Li X, Verma R, Vanzin GF, Caplan J, Shoue DA, Carpita NC, Reiter W-D. The MUR3 gene of Arabidopsis encodes a xyloglucan galactosyltransferase that is evolutionarily related to animal exostosins. Plant Cell. 2003;15:1662.

44. Jensen JK, Schultink A, Keegstra K, Wilkerson CG, Pauly M. RNA-Seq analysis of developing nasturtium seeds (Tropaeolum majus): identification and characterization of an additional galactosyltransferase involved in xyloglucan biosynthesis. Mol Plant. 2012;5:984.

45. Kierzkowski D, Nakayama N, Routier-Kierzkowska AL, Weber A, Bayer E, Schorderet M, Reinhardt D, Kuhlemeier C, Smith RS. Elastic domains regulate growth and organogenesis in the plant shoot apical meristem. Science. 2012;335:1096.

46. Peaucelle A, Louvet R, Johansen JN, Hofte H, Laufs P, Pelloux J, Mouille G. Arabidopsis phyllotaxis is controlled by the methyl-esterification status of cell-wall pectins. Curr Biol. 2008;18:1943.

47. Peaucelle A, Braybrook SA, Le Guillou L, Bron E, Kuhlemeier C, Hofte H. Pectin-induced changes in cell wall mechanics underlie organ initiation in Arabidopsis. Curr Biol. 2011;21:1720.

48. Boavida LC, McCormick S. Temperature as a determinant factor for increased and reproducible in vitro pollen germination in Arabidopsis thaliana. Plant J. 2007;52:570.

49. Bombarely A, Menda N, Tecle IY, Buels RM, Strickler S, Fischer-York T, Pujar A, Leto J, Gosselin J, Mueller LA. The Sol Genomics Network (solgenomics.net): growing tomatoes using Perl. Nucleic Acids Res. 2011;39:D1149.

50. Stonebloom S, Burch-Smith T, Kim I, Meinke D, Mindrinos M, Zambryski P. Loss of the plant DEAD-box protein ISE1 leads to defective mitochondria and increased cell-to-cell transport via plasmodesmata. Proc Natl Acad Sci. 2009;106:17229.

51. Dereeper A, Guignon V, Blanc G, Audic S, Buffet S, Chevenet F, Dufayard JF, Guindon S, Lefort V, Lescot M, Claverie JM, Gascuel O. Phylogeny.fr: robust phylogenetic analysis for the non-specialist. Nucleic Acids Res. 2008;36:W465.

52. Nakagawa T, Kurose T, Hino T, Tanaka K, Kawamukai M, Niwa Y, Toyooka K, Matsuoka K, Jinbo T, Kimura T. Development of series of gateway binary vectors, pGWBs, for realizing efficient construction of fusion genes for plant transformation. J Biosci Bioeng. 2007;104:34.

53. Clough SJ, Bent AF. Floral dip: a simplified method for Agrobacteriummediated transformation of Arabidopsis thaliana. Plant J. 1998;16:735.

54. Sparkes IA, Runions J, Kearns A, Hawes C. Rapid, transient expression of fluorescent fusion proteins in tobacco plants and generation of stably transformed plants. Nat Protoc. 2006;1:2019.

55. Bradford MM. A rapid and sensitive method for the quantitation of microgram quantities of protein utilizing the principle of protein-dye binding. Anal Biochem. 1976;72:248.

56. Eudes A, Baidoo EE, Yang F, Burd H, Hadi MZ, Collins FW, Keasling JD, Loqué $\mathrm{D}$. Production of tranilast [N-(3,4'-dimethoxycinnamoyl)-anthranilic acid] and its analogs in yeast Saccharomyces cerevisiae. Appl Microbiol Biotechnol. 2011:89:989.

57. Czechowski T, Stitt M, Altmann T, Udvardi MK, Scheible WR. Genome-wide identification and testing of superior reference genes for transcript normalization in Arabidopsis. Plant Physiol. 2005;139:5.

58. Vandesompele J, De Preter K, Pattyn F, Poppe B, Van Roy N, De Paepe A, Speleman F. Accurate normalization of real-time quantitative RT-PCR data by geometric averaging of multiple internal control genes. Genome Biol. 2002;3:research0034.

59. OBro J, Harholt J, Scheller HV, Orfila C. Rhamnogalacturonan I in Solanum tuberosum tubers contains complex arabinogalactan structures. Phytochemistry. 2004;65:1429.

60. Chiniquy D, Sharma V, Schultink A, Baidoo EE, Rautengarten C, Cheng K, Carroll A, Ulvskov P, Harholt J, Keasling JD, Pauly M, Scheller HV, Ronald PC. XAX1 from glycosyltransferase family 61 mediates xylosyltransfer to rice xylan. Proc Natl Acad Sci U S A. 2012;109:17117.

61. Pattathil S, Avci U, Miller JS, Hahn MG. Immunological approaches to plant cell wall and biomass characterization: Glycome Profiling. Methods Mol Biol. 2012;908:61.

62. Ciucanu I. Per-O-methylation reaction for structural analysis of carbohydrates by mass spectrometry. Anal Chim Acta. 2006;576:147.

\section{Submit your next manuscript to BioMed Central and we will help you at every step:}

- We accept pre-submission inquiries

- Our selector tool helps you to find the most relevant journal

- We provide round the clock customer support

- Convenient online submission

- Thorough peer review

- Inclusion in PubMed and all major indexing services

- Maximum visibility for your research

Submit your manuscript at www.biomedcentral.com/submit

) Biomed Central 